\title{
Modeling the pressure Hessian and viscous Laplacian in turbulence: Comparisons with direct numerical simulation and implications on velocity gradient dynamics
}

\author{
Citation for published version (APA): \\ Chevillard, L., Meneveau, C., Biferale, L., \& Toschi, F. (2008). Modeling the pressure Hessian and viscous \\ Laplacian in turbulence: Comparisons with direct numerical simulation and implications on velocity gradient \\ dynamics. Physics of Fluids, 20(10), 101504-1/15. [101504]. https://doi.org/10.1063/1.3005832
}

DOI:

10.1063/1.3005832

Document status and date:

Published: 01/01/2008

\section{Document Version:}

Publisher's PDF, also known as Version of Record (includes final page, issue and volume numbers)

\section{Please check the document version of this publication:}

- A submitted manuscript is the version of the article upon submission and before peer-review. There can be important differences between the submitted version and the official published version of record. People interested in the research are advised to contact the author for the final version of the publication, or visit the DOI to the publisher's website.

- The final author version and the galley proof are versions of the publication after peer review.

- The final published version features the final layout of the paper including the volume, issue and page numbers.

Link to publication

\footnotetext{
General rights

- You may freely distribute the URL identifying the publication in the public portal. follow below link for the End User Agreement:

www.tue.nl/taverne

\section{Take down policy}

If you believe that this document breaches copyright please contact us at:

openaccess@tue.nl

providing details and we will investigate your claim.
}

Copyright and moral rights for the publications made accessible in the public portal are retained by the authors and/or other copyright owners and it is a condition of accessing publications that users recognise and abide by the legal requirements associated with these rights.

- Users may download and print one copy of any publication from the public portal for the purpose of private study or research.

- You may not further distribute the material or use it for any profit-making activity or commercial gain

If the publication is distributed under the terms of Article $25 \mathrm{fa}$ of the Dutch Copyright Act, indicated by the "Taverne" license above, please 


\title{
Modeling the pressure Hessian and viscous Laplacian in turbulence: Comparisons with direct numerical simulation and implications on velocity gradient dynamics
}

\author{
L. Chevillard, ${ }^{1,2}$ C. Meneveau, ${ }^{1}$ L. Biferale ${ }^{3}$ and F. Toschi ${ }^{4}$ \\ ${ }^{1}$ Department of Mechanical Engineering and Center for Environmental and Applied Fluid Mechanics, \\ The Johns Hopkins University, 3400 N. Charles Street, Baltimore, Maryland 21218, USA \\ ${ }^{2}$ Laboratoire de Physique de l'École Normale Supérieure de Lyon, CNRS, Université de Lyon, \\ 46 allée d'Italie, F-69007 Lyon, France \\ ${ }^{3}$ Dipartimento di Fisica and INFN, Università di Roma "Tor Vergata," Via della Ricerca Scientifica 1, \\ 00133 Roma, Italy \\ ${ }^{4}$ Department of Physics and Department of Mathematics and Computer Science, \\ Eindhoven University of Technology, P.O. Box 513, 5600 MB Eindhoven, The Netherlands; \\ Istituto per le Applicazioni del Calcolo CNR, 00161 Roma, Italy; and INFN, Sezione di Ferrara, \\ 41000 Ferrara, Italy
}

(Received 4 December 2007; accepted 13 April 2008; published online 31 October 2008)

\begin{abstract}
Modeling the velocity gradient tensor $\mathbf{A}=\boldsymbol{\nabla} \mathbf{u}$ along Lagrangian trajectories in turbulent flow requires closures for the pressure Hessian and viscous Laplacian of A. Based on an EulerianLagrangian change in variables and the so-called recent fluid deformation closure, such models were proposed recently [Chevillard and Meneveau, Phys. Rev. Lett. 97, 174501 (2006)]. The resulting stochastic model was shown to reproduce many geometric and anomalous scaling properties of turbulence. In this work, direct comparisons between model predictions and direct numerical simulation (DNS) data are presented. First, statistical properties of $\mathbf{A}$ are described using conditional averages of strain skewness, enstrophy production, energy transfer, and vorticity alignments, conditioned upon invariants of the velocity gradient. These conditionally averaged quantities are found to be described accurately by the stochastic model. More detailed comparisons that focus directly on the terms being modeled in the closures are also presented. Specifically, conditional statistics associated with the pressure Hessian and the viscous Laplacian are measured from the model and are compared with DNS. Good agreement is found in strain-dominated regions. However, some features of the pressure Hessian linked to rotation-dominated regions are not reproduced accurately by the model. Geometric properties such as vorticity alignment with respect to principal axes of the pressure Hessian are mostly predicted well. In particular, the model predicts that an eigenvector of the rate of strain will be also an eigenvector of the pressure Hessian, in accord with basic properties of the Euler equations. The analysis identifies under what conditions the Eulerian-Lagrangian change in variables with the recent fluid deformation closure works well, and in which flow regimes it requires further improvements. () 2008 American Institute of Physics.
\end{abstract}

[DOI: $10.1063 / 1.3005832]$

\section{INTRODUCTION}

Fundamental understanding of universal features of the small-scale structure of turbulence has been a long-standing challenge in turbulence research. ${ }^{1-7}$ While considerable phenomenological understanding has been accumulated in recent decades, the challenge of relating observed phenomena and statistical properties to the dynamical equations (NavierStokes) remains unmet. The velocity gradient tensor $A_{i j}$ $=\partial u_{i} / \partial x_{j}$ (where $\mathbf{u}$ denotes the velocity vector) provides a rich characterization of the topological and statistical properties of the fine-scale structures in turbulence. Having a spectral peak at around the Kolmogorov wavelength $k_{\eta}$ $\sim \eta^{-1}$ ( $\eta$ is the Kolmogorov dissipative length scale), $A_{i j}$ is a quantity dominated by motions in the viscous range. The antisymmetric part of the tensor is the vorticity representing small-scale rotation of fluid elements, while its symmetric part, the strain-rate tensor, represents fluid deformation rate. The Lagrangian evolution of this tensor can be described by an evolution equation that is obtained from taking the gradient of the Navier-Stokes equations. The resulting system is unclosed since it contains the anisotropic part of the pressure Hessian and the viscous term. When both of these are neglected, the system is closed [called restricted Euler (RE) dynamics $].^{8-11}$ The RE equations already predict several known geometric turbulence phenomena associated with $A_{i j},{ }^{12-20}$ such as preferential alignments of vorticity with strain-rate eigenvectors and preponderance of axisymmetric expansion and positiveness of the intermediate eigenvalue of the strain rate. Nevertheless, RE produces singularities in a relatively short, finite time (see Ref. 7 for a review).

Phenomenologically, other phenomena such as smallscale intermittency may also be probed, by studying the probability distribution functions (PDFs) of individual velocity gradient elements. For instance, it is known that the PDFs of longitudinal and transverse gradients, e.g., $A_{11}$ and $A_{12}$, in particular directions, respectively, can be described by elongated stretched exponential tails $\mathrm{s}^{5,21-23}$ or by superposition of 
stretched exponential. ${ }^{24}$ Also the moments of these gradients scale in nontrivial (anomalous) ways with the Reynolds number. ${ }^{25,26}$ Understanding such anomalous scaling behavior of turbulence is another open challenge. Therefore, the wealth of geometric, dynamical, and statistical turbulence phenomena that can be described by the velocity gradient tensor, coupled with the fact that a dynamical equation (even though unclosed) is available from the gradient of the Navier-Stokes equations, makes $A_{i j}$ a tensor variable of considerable interest for further study. The role of pressure in the intermittent nature of velocity gradients was also pointed out by Kraichnan in early works. ${ }^{27,28}$

Based on prior works, ${ }^{10,11,29-33}$ a stochastic dynamical model for the time evolution of $A_{i j}$ has been proposed. ${ }^{34,35}$ The model includes a closure for the pressure Hessian, i.e., $\partial^{2} p / \partial x_{i} \partial x_{j}$, and the viscous term, i.e., $\nu \nabla^{2} \mathbf{A}$ in terms of the local value of the velocity gradient tensor. The approach, reviewed in detail in Sec. II, consists in a change in variables from Eulerian positions to Lagrangian labels before assuming isotropy in the associated gradient tensors to be modeled. The Eulerian-Lagrangian transformation involves a Jacobian matrix that is modeled using the local value of the velocity gradient tensor by using the "recent fluid deformation" closure. The model system is forced using a Gaussian white-intime random force. The resulting stochastic model consists of eight independent coupled stochastic differential equations (SDEs) that aim to describe the time evolution of each of the tensor elements of $A_{i j}$, following a fluid particle in a turbulent flow.

The results of Ref. 34 show that the finite-time divergence exhibited by the RE system is regularized with the inclusion of the proposed models for pressure Hessian and viscous term. Moreover, with the random forcing, stationary statistics of the velocity gradient tensor are obtained, with realistic statistical properties such as preferential alignment of vorticity and the preferential state of axisymmetric expansion. The shape of PDFs of longitudinal and transverse gradients is quite realistic and even some well-known properties of anomalous scaling in turbulence are reproduced. ${ }^{34,35} \mathrm{~A}$ limitation of the model is that at high Reynolds numbers the resulting distribution functions became increasingly unrealistic. One approach to remedy this problem has been explored ${ }^{36}$ by constructing a multiscale version of the model, i.e., a matrix shell model that describes the velocity gradient tensors in various shells at different scales. The closure for the interscale interaction terms is based on the criterion that the total kinetic energy must be preserved by the modeled quadratic interscale interaction terms. The introduction of nonlocal (in scale) interactions leads to a structure of the model that is more difficult to analyze theoretically, but it provides an interesting connection between the gradients' evolution at various scales and the energy cascade mechanism. While the matrix shell model successfully eliminates the problems at high Reynolds numbers, it does not make an explicit connection with the physics of the pressure Hessian. At this stage, then, it is of interest to further improve our understanding of the fundamental properties of the closures proposed in Ref. 34, developed from the expression of pressure Hessian and viscous term as given from the Navier-
Stokes equations, within the range of Reynolds numbers in which the model of Ref. 34 works well.

In Sec. III various model predictions of statistical and geometric properties of $\mathbf{A}$ beyond those already studied in Ref. 34 are compared with direct numerical simulation (DNS) at a moderate Reynolds number $\left(\mathcal{R}_{\lambda}=150\right)$. The model is evaluated using statistical measures already studied in Ref. 30 in the context of the "tetrad model." These measures include conditional averages of the "dissipation" $\left(|\mathbf{S}|^{2}\right.$ $=S_{i j} S_{i j}$, where $\mathbf{S}$ is the symmetric part of $\left.\mathbf{A}\right)$ and of the "enstrophy" (i.e., $|\boldsymbol{\Omega}|^{2}=\Omega_{i j} \Omega_{i j}$, where $\boldsymbol{\Omega}$ is the antisymmetric part of $\mathbf{A}$ ). Then, a similar analysis is performed with the enstrophy production and the strain skewness. The conditional averages are expressed in terms of the two principal invariants of $\mathbf{A}$, namely $R=-(1 / 3) \operatorname{Tr}\left[\mathbf{A}^{3}\right]$ and $Q$ $=-(1 / 2) \operatorname{Tr}\left[\mathbf{A}^{2}\right]$. Different regions in the " $(R, Q)$-plane" have distinct physical interpretations ${ }^{11,13,15,37}$ and the behavior of the computed conditional averages in these different regions thus provide useful and statistically meaningful insights into the performance of the model in dynamically very different regions of the flow.

In order to quantify and understand the average local evolution of the turbulence dynamics in the $(R, Q)$-plane, the probability current of the joint probability density $\mathcal{P}(Q, R)$ is also studied in Sec. IV. These statistics depend explicitly on both pressure Hessian and viscous Laplacian, and thus the effect of the proposed closures for these terms may be compared with the real effects obtained from the DNS.

In Sec. V the preferential alignment of vorticity with eigendirections of both pressure Hessian and the symmetric part of the viscous Laplacian are studied in detail. Connections with theoretical results pertaining to the Euler equations are also made. Finally, in Sec. VI the results are summarized and conclusions are presented.

\section{THEORETICAL BACKGROUND}

\section{A. Lagrangian description of the velocity gradient tensor}

A description of small-scale structure of turbulence based on the velocity gradient tensor $A_{i j}=\partial u_{i} / \partial x_{j}$ begins by taking the gradient of the Navier-Stokes equation. One then obtains the system

$$
\frac{d A_{i j}}{d t}=-A_{i k} A_{k j}-\frac{\partial^{2} p}{\partial x_{i} \partial x_{j}}+\nu \frac{\partial^{2} A_{i j}}{\partial x_{k} \partial x_{k}},
$$

where $d / d t$ stands for the Lagrangian material derivative (i.e., $d / d t \equiv \partial / \partial t+u_{k} \partial / \partial x_{k}$ ), $p$ is the pressure divided by the density of the fluid, and $\nu$ is the kinematic viscosity. Because of incompressibility, $\mathbf{A}$ must remain trace-free, i.e., $A_{i i}=0$. Equation (1) is not closed in terms of $\mathbf{A}$ at the position $\mathbf{x}$ and time $t$. This can be easily seen noting that the pressure field is the solution of the Poisson equation $\operatorname{Tr}\left[\partial^{2} p / \partial x_{i} \partial x_{j}\right]=\nabla^{2} p$ $=-A_{l k} A_{k l}$ which shows that pressure is highly nonlocal. Moreover the viscous term requires the Laplacian of $\mathbf{A}$ which is not known simply in terms of $\mathbf{A}$.

As already mentioned in Sec. I, neglecting pressure Hessian anisotropy and viscous effects leads to finite-time singularities because of the strong and unopposed effects of the 
self-stretching term $-\mathbf{A}^{2}$. One can find in the literature several efforts at regularizing this finite-time divergence, while keeping the exact self-stretching term. First, Girimaji and Pope ${ }^{29}$ succeeded to do so by constructing a stochastic model with an imposed constraint. This constraint is imposed by modifying the nonlinear term so that the pseudodissipation $\varphi=A_{i j} A_{i j}$ (Ref. 38) is lognormal with a prescribed mean and variance. Intermittency trends are put in explicitly, by prescribing a known variance of $\log (\varphi)$ as function of the Reynolds number.

More recently, two groups proposed the idea that the local geometry of the accumulated fluid deformation, i.e., formally the Cauchy-Green tensor, may represent the missing information which allows to regularize the RE divergence. Accumulated fluid deformation thus has been used to model the pressure Hessian in the so-called tetrad model of Pumir and co-workers. ${ }^{30,39,40}$ A similar idea from Jeong and Girimaji ${ }^{31}$ has been used to model the viscous part of Eq. (1), explicitly using the Cauchy-Green tensor. Whether or not the finite-time divergence is regularized, the direct use of the Cauchy-Green tensor is limited by the fact that this tensor is fundamentally nonstationary, i.e., as time evolves it maintains memory of the initial condition. Hence the resultant models for pressure Hessian and viscous term are intrinsically nonstationary and depend on the initial condition chosen to initialize the material deformation tracking. In the following sections, we discuss these issues in more detail and also review the simplified model of Ref. 34 that avoids these problems of nonstationarity.

We also point out an alternative approach ${ }^{41}$ that renormalizes the time variable in RE dynamics so as to relegate the finite-time singularities to infinite time.

\section{B. Lagrangian mapping and Cauchy-Green tensor}

Following Refs. 4, 42, and 43, one may define a mapping $\mathcal{T}_{t_{0}, t}$ between Eulerian and Lagrangian coordinates,

$$
\mathcal{T}_{t_{0}, t}: \mathbf{X} \in \mathbb{R}^{3} \mapsto \mathbf{x} \in \mathbb{R}^{3},
$$

where $\mathbf{x}(\mathbf{X}, t)$ denotes the position at a time $t$ of a fluid particle which was at the position $\mathbf{x}\left(\mathbf{X}, t_{0}\right)=\mathbf{X}$ at the initial time $t_{0}$. Given the initial position of a fluid particle, this mapping [Eq. (2)] is fully defined at any time by

$$
\frac{d \mathbf{x}}{d t}=\mathbf{u}(\mathbf{x}, t) .
$$

A quantity of much interest in continuous mechanics is the deformation gradient tensor $\mathbf{D}$, defined as $D_{i j}=\partial x_{i} / \partial X_{j}$, which relates the variation in the position of a particle when one slightly changes the initial position. Differentiating Eq. (3) with respect to $X_{j}$, one gets the time evolution of $\mathbf{D}$, i.e.,

$$
\frac{d \mathbf{D}}{d t}=\mathbf{A}(t) \mathbf{D}(t),
$$

and one can show ${ }^{4,43}$ that the Jacobian of the mapping $\mathcal{T}_{t_{0}, t}$, i.e., $\operatorname{det}(\mathbf{D}(t))$, is equal to unity at any time by virtue of incompressibility, stating that this mapping is always invertible. Equation (4) can be exactly solved using the product integral $^{44}$ or alternatively, the time-ordered exponential, ${ }^{45,46}$

$$
\mathbf{D}=\prod_{t_{0}}^{t} e^{d s \mathbf{A}(s)}=\mathcal{T}^{+} \exp \left[\int_{t_{0}}^{t} d s \mathbf{A}(s)\right] .
$$

The Cauchy-Green tensor $\mathbf{C}(t)$ is defined as the symmetric tensor $\mathbf{C}=\mathbf{D D}^{\top}$ and its eigenvalue and eigenvector system describes the rotation and deformation of initially isotropicshaped fluid volumes into various shapes as time goes on. The transport equation of the Cauchy-Green tensor can be obtained in straightforward fashion ${ }^{47}$ from Eq. (4),

$$
\frac{d \mathbf{C}}{d t}=\mathbf{A}(t) \mathbf{C}(t)+\mathbf{C}(t) \mathbf{A}^{\top}(t) .
$$

Based on the properties of $\mathbf{C}$, studies of isotropic and homogeneous turbulence in both numerical ${ }^{48}$ and laboratory ${ }^{7,19}$ flows have shown that cigar (one large and two small eigenvalues of $\mathbf{C}$ ) and pancake (two large and one small eigenvalue) shapes are the most common shapes of fluid deformation.

\section{Fluid deformation and pressure Hessian models}

Let us first remark that the pressure Hessian is not among the most studied objects in the turbulence literature (although, see Ref. 49). One reason perhaps is that it cannot be described naturally from a standard transport equation along a Lagrangian trajectory. Instead, the pressure Hessian is related to the spatial distribution of the velocity gradient using singular integral operators, ${ }^{43,50,51}$

$$
\frac{\partial^{2} p}{\partial x_{i} \partial x_{j}}=-\operatorname{Tr}\left(\mathbf{A}^{2}\right) \frac{\delta_{i j}}{3}-\text { P.V. } \int k_{i j}(\mathbf{x}-\mathbf{y}) \operatorname{Tr}\left(\mathbf{A}^{2}\right)(\mathbf{y}) d \mathbf{y},
$$

where the integral is understood as a Cauchy principal value and $k_{i j}$ is the Hessian of Laplace's Green's function, namely

$$
k_{i j}(\mathbf{x})=\frac{\partial^{2}}{\partial x_{i} \partial x_{j}} \frac{1}{4 \pi|\mathbf{x}|}=\frac{|\mathbf{x}|^{2} \delta_{i j}-3 x_{i} x_{j}}{4 \pi|\mathbf{x}|^{5}} .
$$

One can see from Eq. (7) that only the isotropic part of the pressure Hessian is purely local [the first term of the right hand side of Eq. (7)]. All the nonlocal effects of pressure Hessian enter through the anisotropic part [or deviatoric part corresponding to the second term of the right hand side of Eq. (7)]. Hence, in this view, the RE approximation can be understood as the neglect of all the nonlocal effects implied by the incompressibility condition: the corresponding Lagrangian particle evolves with the flow completely independent from its neighbors. As far as we know, the tetrad model $^{30}$ is the first model to have been proposed for the anisotropic (i.e., nonlocal) part of the pressure Hessian. While the authors introduced the model using the language of multipoint dispersion of particles that define an evolving tetrad shape, a simple interpretation of the model can also be given in terms of the deformation and Cauchy-Green tensors.

To begin, one can re-express various Eulerian quantities such as the pressure Hessian and the viscous term in terms of Lagrangian coordinates, i.e., in terms of the fluid particle's 
position at some initial time $t_{0}, \mathbf{X}$. For the Hessian tensor of the pressure at the current point and time $(\mathbf{x}, t)$, one may write

$$
\frac{\partial^{2} p(\mathbf{x}, t)}{\partial x_{i} \partial x_{j}}=\frac{\partial X_{p}}{\partial x_{i}} \frac{\partial X_{q}}{\partial x_{j}} \frac{\partial^{2} p(\mathbf{x}, t)}{\partial X_{p} \partial X_{q}}+\frac{\partial^{2} X_{q}}{\partial x_{i} \partial x_{j}} \frac{\partial p(\mathbf{x}, t)}{\partial X_{q}} .
$$

The second term entering in the right hand side of Eq. (9) requires the knowledge of the spatial distribution of the (inverse) deformation gradient, through its spatial derivative. As will be seen later, the adopted approach neglects shorttime variations in the velocity gradient and in the context of the proposed Lagrangian model, it is natural to neglect spatial fluctuations of the deformation gradient, i.e., $\partial^{2} X_{q} / \partial x_{i} \partial x_{j} \sim 0$. Next, we discuss the remaining term of the right hand side of Eq. (9). The fourth-order tensor $\partial_{i} X_{p} \partial_{j} X_{q}$ can be solved along its trajectory using the dynamical evolution for the deformation tensors [Eq. (4)]. For the remaining factor, the Lagrangian pressure Hessian $\partial^{2} p / \partial X_{p} \partial X_{q}$, we choose the simplest assumption, namely the isotropic assumption,

$$
\frac{\partial^{2} p}{\partial X_{p} \partial X_{q}}=\frac{1}{3} \frac{\partial^{2} p}{\partial X_{m} \partial X_{m}} \delta_{p q} .
$$

Physically, this assumption states that as time progresses, one loses memory about the relative orientations of the initial locations $\mathbf{X}$ as far as the present value of pressure is concerned. The contraction between $\delta_{p q}$ and $\partial_{i} X_{p} \partial_{j} X_{q}$ then connects the model to the Cauchy-Green tensor introduced in the preceding section.

So far the pressure Hessian can then be rewritten, using Eq. (10), according to

$$
\frac{\partial^{2} p}{\partial x_{i} \partial x_{j}} \approx \frac{\partial X_{m}}{\partial x_{i}} \frac{\partial X_{n}}{\partial x_{j}} \frac{\partial^{2} p(\mathbf{x}, t)}{\partial X_{m} \partial X_{n}}=C_{i j}^{-1} \frac{1}{3} \frac{\partial^{2} p(\mathbf{x}, t)}{\partial X_{k} \partial X_{k}} .
$$

To determine $\partial^{2} p / \partial X_{k} \partial X_{k}$, we follow Ref. 30 and use the Poisson equation $\nabla^{2} p=-A_{n m} A_{m n}=(1 / 3) C_{q q}^{-1} \partial^{2} p / \partial X_{k} \partial X_{k}$. Replacing back into Eq. (11) leads to ${ }^{30,34}$

$$
\frac{\partial^{2} p(t)}{\partial x_{i} \partial x_{j}}=-\frac{\operatorname{Tr}\left(\mathbf{A}^{2}\right)}{\operatorname{Tr}\left(\mathbf{C}^{-1}\right)} C_{i j}^{-1}=\frac{2 Q}{\operatorname{Tr}\left(\mathbf{C}^{-1}\right)} C_{i j}^{-1} .
$$

\section{Fluid deformation and modeling the viscous Laplacian}

In a similar fashion, following Ref. 31, this procedure can be applied to the viscous Laplacian entering in the gradient of the Navier-Stokes equation [Eq. (1)], i.e.,

$$
\nu \frac{\partial^{2} \mathbf{A}}{\partial x_{k} \partial x_{k}} \approx \frac{\partial X_{p}}{\partial x_{k}} \frac{\partial X_{q}}{\partial x_{k}}\left(\nu \frac{\partial^{2} \mathbf{A}}{\partial X_{p} \partial X_{q}}\right) \text {. }
$$

The resulting Lagrangian Hessian of $\mathbf{A}$ entering in Eq. (13) will be considered as (i) isotropic, i.e., $\partial^{2} \mathbf{A} /\left(\partial X_{p} \partial X_{q}\right)$ $=\partial^{2} \mathbf{A} /\left(\partial X_{m} \partial X_{m}\right) \delta_{p q} / 3$, and (ii) its trace will be modeled by a friction term, i.e., $\partial^{2} \mathbf{A} /\left(\partial X_{m} \partial X_{m}\right)=-1 / \ell^{2} \mathbf{A}$. The characteristic length scale $\ell$ reflects the typical length in the Lagrangian frame over which $\mathbf{A}$ is correlated. To estimate this length scale, we note that the typical decorrelation time of $\mathbf{A}$ along its Lagrangian trajectory is known to be on the order of $\tau_{K}$ $=(\nu / \epsilon)^{1 / 2}$, the Kolmogorov time scale (where $\epsilon$ is the dissipation rate). ${ }^{2}$ During that time, a fluid particle is advected by the turbulence over a distance of the order of $\ell=u^{\prime} \tau_{K}=\lambda$, where $u^{\prime}$ is the root mean square velocity (chosen as advective velocity scale) and $\lambda$ the Taylor microscale. Finally, recognizing that $\nu / \lambda^{2}=T^{-1}$, where $T$ is the integral time scale, one then obtains the following model for the viscous term:

$$
\nu \frac{\partial^{2} \mathbf{A}}{\partial x_{k} \partial x_{k}} \approx-\frac{1}{T} \frac{\operatorname{Tr}\left(\mathbf{C}^{-1}\right)}{3} \mathbf{A} .
$$

This model is similar to the one obtained by Jeong and Girimaji $^{31}$ but using a different, more physically motivated time scale.

\section{E. Stochastic model based on the recent fluid deformation closure}

The various terms entering in the right hand side of Eqs. (12) and (14) include the tensor $\mathbf{C}$. If this tensor is obtained from its transport equation [Eq. (6)] subject to the natural initial condition $C_{i j}\left(t_{0}\right)=\delta_{i j}$, then the closures for pressure Hessian and viscous term depend strongly on the initial time $t_{0}$ or, equivalently, on the initial position $\mathbf{X}$. Due to the dispersive nature of turbulent flow, $\mathbf{C}$ continues to evolve with exponentially growing and decreasing eigenvalues. Instead of solving for $\mathbf{C}$ from its transport equation and having to deal with the problems associated with nonstationarity, in Ref. 34 a simple closure was proposed. It consists of a sort of "Markovianization" of the dynamics of $\mathbf{C}$ in that it is assumed that $\mathbf{C}$ evolves in a frozen velocity gradient tensor field during a characteristic (short) time $\tau$. The value of $\mathbf{A}$ during that time is taken as the most recent value (i.e., the current, local, value). Additionally the time scale chosen is the typical decorrelation time scale of $\mathbf{A}$ during its Lagrangian evolution, which is known to be of the order of the Kolmogorov time scale $\tau_{K}$. Thus, the initial time is taken to be at $t_{0}=t-\tau_{K}$, which allows to write in a simple way the timeordered exponential entering in Eq. (5). We thus replace the true Cauchy-Green tensor by a new tensor, called the "recent Cauchy-Green tensor" $\mathbf{C}_{\tau_{K}}$ that can be expressed in terms of simple matrix exponentials,

$$
\mathbf{C}_{\tau_{K}}=e^{\tau_{K} \mathbf{A}} e^{\tau_{K} \mathbf{A}^{\top}}
$$

This leads to an explicit A-dependent model for the full pressure Hessian

$$
\frac{\partial^{2} p}{\partial x_{i} \partial x_{j}}=-\frac{\operatorname{Tr}\left(\mathbf{A}^{2}\right)}{\operatorname{Tr}\left(\mathbf{C}_{\tau_{K}}^{-1}\right)}\left(\mathbf{C}_{\tau_{K}}^{-1}\right)_{i j}
$$

and for the viscous Laplacian

$$
\nu \frac{\partial^{2} \mathbf{A}}{\partial x_{k} \partial x_{k}}=-\frac{1}{T} \frac{\operatorname{Tr}\left(\mathbf{C}_{\tau_{K}}^{-1}\right)}{3} \mathbf{A} .
$$

Inserting Eqs. (16) and (14) into Eq. (1) and writing the equation in the Itô's language of SDEs, ${ }^{52}$ the full model for the time evolution of the velocity gradient reads 


$$
d \mathbf{A}=\left[-\mathbf{A}^{2}+\frac{\operatorname{Tr}\left(\mathbf{A}^{2}\right)}{\operatorname{Tr}\left(\mathbf{C}_{\tau_{K}}^{-1}\right)} \mathbf{C}_{\tau_{K}}^{-1}-\frac{\operatorname{Tr}\left(\mathbf{C}_{\tau_{K}}^{-1}\right)}{3 T} \mathbf{A}\right] d t+d \mathbf{W} .
$$

The stochastic time evolution of the velocity gradient tensor A [Eq. (18)], as proposed in Refs. 34 and 35, relates the joint deterministic action of the self-stretching term $-\mathbf{A}^{2}$, the pressure Hessian [Eq. (16)], and the viscous term [Eq. (17)]. Moreover, the system is forced with a stochastic Gaussian noise. The deterministic part provides two time scales: a small time scale $\tau_{K}$ and a large one $T$. The latter arises in modeling the viscous diffusion term when combining the viscosity with the Taylor microscale, which in turn is related to the large-scale velocity rms. Hence, the deterministic part gives the dependence on the Reynolds number Re to the model through the ratio $\left(T / \tau_{K}\right) \sim \operatorname{Re}^{1 / 2}$, according to classical Kolmogorov dimensional arguments. ${ }^{5}$ Dependence on the Reynolds number of higher order moments of velocity derivatives (i.e., anomalous scalings and the intermittency phenomenon) has been studied and quantified in Ref. 35. The purpose of this article is to focus on a single Reynolds number and to compare it with a DNS flow (see next paragraph).

The term $\mathbf{W}$ is a tensorial delta-correlated noise term that has been added in order to represent possible forcing effects, e.g., from neighboring eddies. ${ }^{34,35}$ In Appendix A, we describe this noise extensively and propose a way to simulate it.

\section{F. DNS data and comparisons with the model}

In the following, we will make extensive use of a standard DNS of the Navier-Stokes equation for a Taylor based Reynolds number of order $\mathcal{R}_{\lambda}=150$. Pseudospectral simulations are performed, of an isotropic turbulent flow in a $[0,2 \pi]^{3}$ box using $256^{3}$ nodes. Fourier modes in shells with $|\mathbf{k}|<2$ are forced by a term added to the Navier-Stokes equations, which provides constant energy injection rate $\epsilon_{f}$ $=0.1$. The viscosity of the fluid is $\nu=0.00113$. The time step $\Delta t$ is chosen adaptively to ensure the Courant number $\Delta t u_{\max } / \Delta x \leq 0.15$, where $u_{\max }$ is the maximum velocity and $\Delta x$ is the grid size. In order to make comparisons between DNS data and the model, one has to specify a value for the parameter of the model $\tau_{K}$. At $\mathcal{R}_{\lambda}=150$, it has been estimated by Yeung et $a .^{53}$ that the ratio of the Kolmogorov scale and the integral (i.e., velocity correlation time scale) time scale is $\tau_{K} / T \approx 0.1$. Thus, in the following, DNS data will be compared to the model run with $\tau_{K}=0.1 \mathrm{~T}$. Without loss of generality, the integral time scale $T$ will be set to unity. It corresponds to set time as units of $T$. The model as written out as in Eq. (18) is solved numerically, with the parameter $\tau_{K}$ $=0.1$, using a second-order predictor-corrector method (see Ref. 52) with a time step of $\Delta t=10^{-3}$. One obtains time series of all of the components of the tensor A that display temporally stationary statistics. In this article, we have worked with a time series of length of $\sim 10^{6}$ in units of the integral time scale $T$. These can then be directly compared to DNS results. Furthermore the model provides statistically stationary time series for both pressure Hessian and viscous Laplacian that can be also directly compared to DNS data.

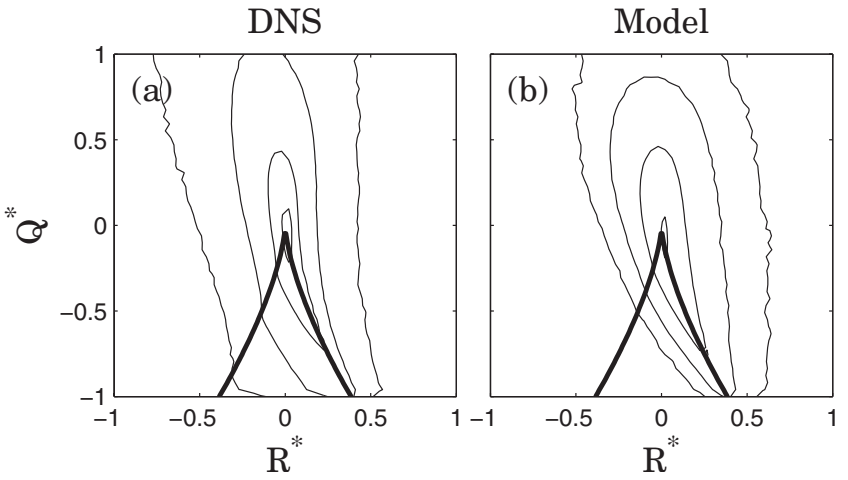

FIG. 1. Joint PDF $\mathcal{P}\left(Q^{*}, R^{*}\right)$ of $R^{*}=R /\left\langle S_{i j} S_{i j}\right\rangle^{3 / 2}$ and $Q^{*}=Q /\left\langle S_{i j} S_{i j}\right\rangle$ calculated from DNS (a) and the present model (b). Contour lines are the same in the two cases, logarithmically spaced by a factor of 10 , and start at 10 near the origin. The thick line represents the zero-discriminant (or Vieillefosse) line: $\frac{27}{4} R^{2}+Q^{3}=0$.

\section{CONDITIONAL STATISTICS OF THE VELOCITY GRADIENT TENSOR}

We are interested here in studying various properties of the velocity gradient tensor conditioned upon the value of its two invariants $R$ and $Q$ defined earlier. The joint probability density of $(R, Q)$ (the $R Q$-plane) has been studied in the past $^{10,11,13,15,37}$ and can be used to characterize the frequency of occurrence of the various local topologies of the flow.

For instance, in a simple way, the second invariant

$$
Q=-\frac{1}{2} \operatorname{Tr}\left(\mathbf{A}^{2}\right)=\frac{1}{4}|\omega|^{2}-\frac{1}{2} \operatorname{Tr}\left(\mathbf{S}^{2}\right)
$$

can be understood as the competition between enstrophy ( $\omega$ denotes vorticity) and dissipation (per unit viscosity). Then, positive $Q$ represents rotation-dominated regions and negative $Q$ dissipation-dominated regions. Analogously, the third invariant

$$
R=-\frac{1}{3} \operatorname{Tr}\left(\mathbf{A}^{3}\right)=-\frac{1}{4} \omega_{i} S_{i j} \omega_{j}-\frac{1}{3} \operatorname{Tr}\left(\mathbf{S}^{3}\right)
$$

represents competition between enstrophy production entering in the enstrophy evolution, ${ }^{7,54}$ i.e.,

$$
\frac{1}{2} \frac{d|\omega|^{2}}{d t}=\omega_{i} S_{i j} \omega_{j}+\nu \omega_{i} \nabla^{2} \omega_{i},
$$

and the dissipation production (or strain skewness ${ }^{30}$ ) entering in the dissipation evolution, i.e.,

$$
\begin{aligned}
\frac{d \operatorname{Tr}\left(\mathbf{S}^{2}\right)}{d t}= & -2 \operatorname{Tr}\left(\mathbf{S}^{3}\right)-\frac{1}{2} \omega_{i} S_{i j} \omega_{j}-2 S_{i j} \frac{\partial^{2} p}{\partial x_{i} \partial x_{j}} \\
& +\nu S_{i j} \nabla^{2} S_{i j} .
\end{aligned}
$$

Let us remark that one may interpret the $R Q$ plane in a different way, based on the eigenvalues of $\mathbf{A}$ (two of them can be the complex conjugate) and the zero-discriminant line (i.e., the "Vieillefosse" line, namely $\frac{27}{4} R^{2}+Q^{3}=0$ ). See, for instance, Refs. 11, 13, and 37.

\section{A. The joint PDF in the RQ-plane}

We show in Fig. 1 the joint PDF of $R$ and $Q$ or, equivalently, the joint $\operatorname{PDF} \mathcal{P}\left(Q^{*}, R^{*}\right)$ of the nondimensionalized invariants $R^{*}=R /\left\langle S_{i j} S_{i j}\right\rangle^{3 / 2}$ and $Q^{*}=Q /\left\langle S_{i j} S_{i j}\right\rangle$ for both DNS 

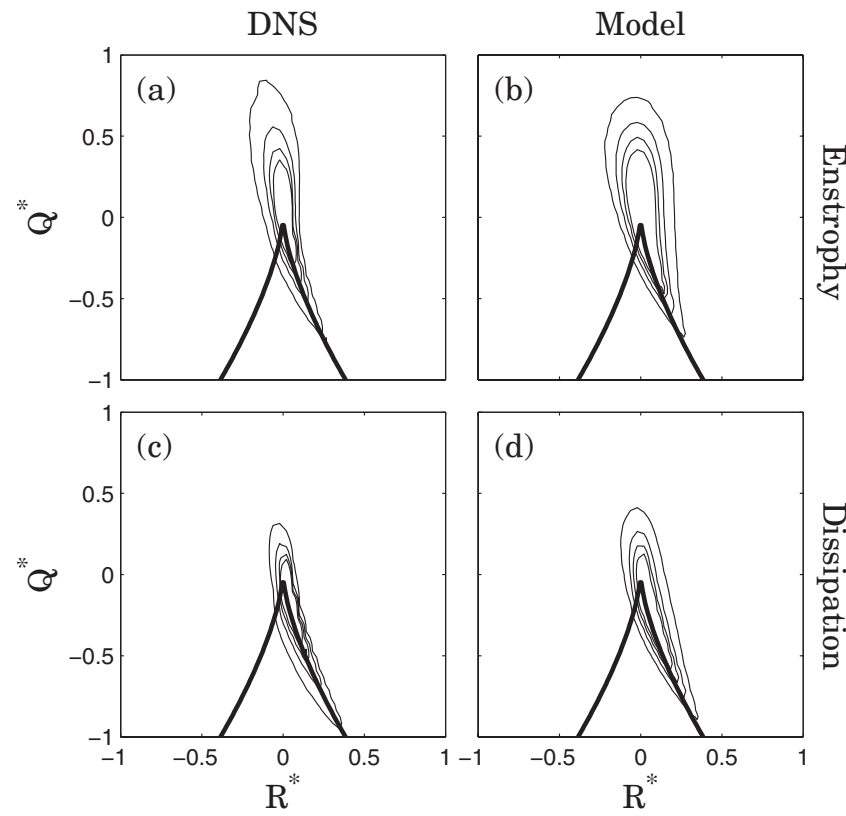

FIG. 2. Isocontours of the conditional enstrophy $\left\langle\omega_{i} \omega_{i} \mid Q^{*}, R^{*}\right\rangle \mathcal{P}\left(Q^{*}, R^{*}\right)$, where $\boldsymbol{\omega}$ is the vorticity, and conditional dissipation $\left\langle S_{i j} S_{i j} \mid Q^{*}, R^{*}\right\rangle \mathcal{P}\left(Q^{*}, R^{*}\right)$. Following Ref. 30, both quantities are normalized by $\left\langle 2 \Omega_{i j} \Omega_{i j}\right\rangle$, where $\boldsymbol{\Omega}$ is the rate of rotation tensor. Level of contour lines are $0.15,0.3,0.45,1,2,3$, and 4 .

and the model [Eq. (18)]. In order to compute various conditional averages, a range $[-1 ; 1]$ of values of the two nondimensionalized invariants $R^{*}$ and $Q^{*}$ of $\mathbf{A}$ is discretized in 25 equally spaced bins. For the DNS, one observes the predominance of the enstrophy-enstrophy production quadrant $\left(R^{*}<0\right.$ and $\left.Q^{*}>0\right)$ and the dissipation-dissipation production quadrant $\left(R^{*}>0\right.$ and $\left.Q^{*}<0\right)$. The predominance of these two quadrants has been observed before in the literature. ${ }^{13}$ The model reproduces these basic trends fairly accurately, with the characteristic "tear-shape" elongation along the "Vieillefosse tail" in the dissipation-dissipation production quadrant. But one also observes that the model overestimates the total probability in the enstrophydissipation production region (i.e., $R^{*}>0$ and $Q^{*}>0$ ) and underestimates the dissipation-enstrophy production region (i.e., $R^{*}<0$ and $Q^{*}<0$ ). It will be shown later that this is caused by limitations in how the pressure Hessian is closed and modeled.

Next, we check whether or not dissipation (enstrophy) is dominantly associated with the $R^{*}>0$ and $Q^{*}<0 \quad\left(R^{*}<0\right.$ and $Q^{*}>0$ ) quadrants. Following the approach already used in Ref. 30 we present in Fig. 2 the conditional averages of dissipation, i.e., $\left\langle\operatorname{Tr}\left(\mathbf{S}^{2}\right) \mid Q^{*}, R^{*}\right\rangle \mathcal{P}\left(Q^{*}, R^{*}\right)$ and enstrophy $\left\langle\omega_{i} \omega_{i} \mid Q^{*}, R^{*}\right\rangle \mathcal{P}\left(Q^{*}, R^{*}\right)$. Averages are weighted by the joint density $\mathcal{P}\left(Q^{*}, R^{*}\right)$ to ensure that the sum over all possible values of $R^{*}$ and $Q^{*}$ gives the averages of, respectively, dissipation and enstrophy. We clearly see that the quadrants $R^{*}>0$ and $Q^{*}<0$ (or $R^{*}<0$ and $Q^{*}>0$ ) are dominated by dissipation (or enstrophy). The model reproduces these conditional averages quite accurately.
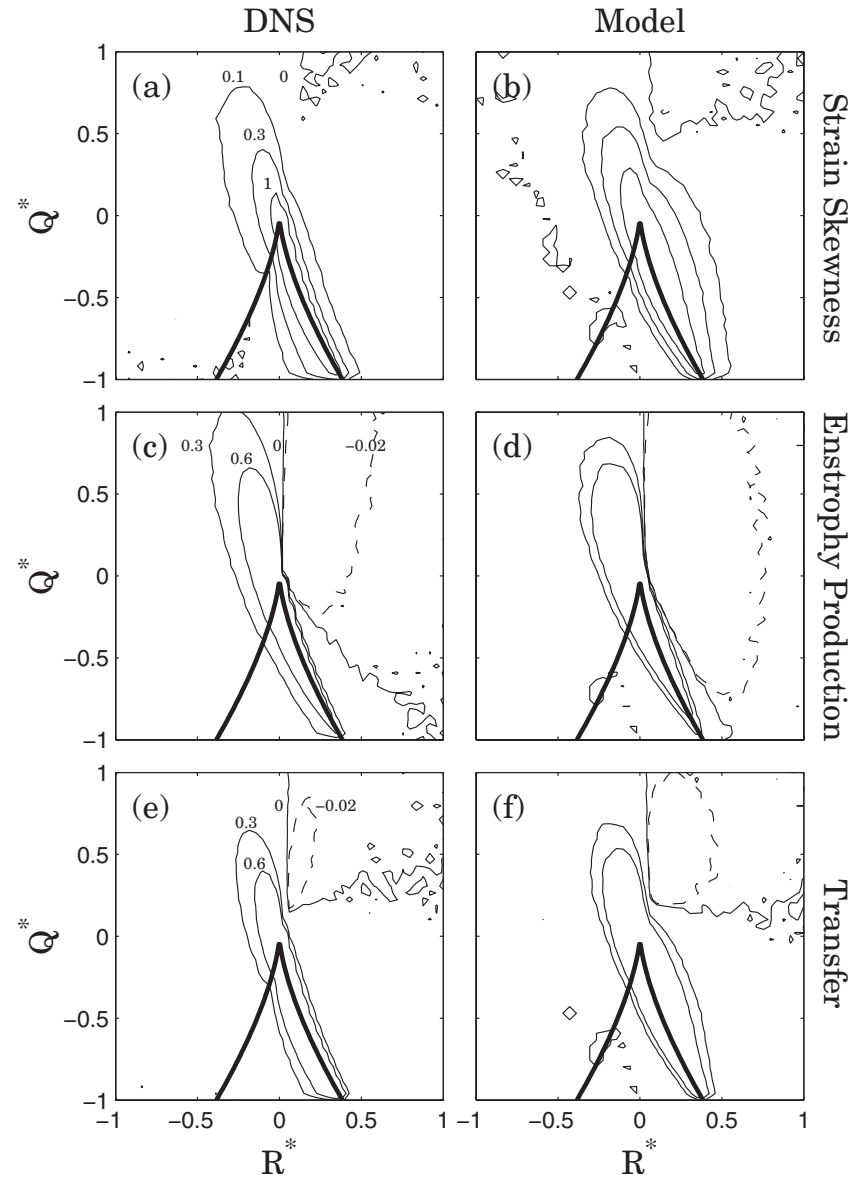

FIG. 3. Isocontours of the conditional strain skewness $-\left\langle\operatorname{Tr}\left(\mathbf{S}^{3}\right) \mid Q^{*}, R^{*}\right\rangle \mathcal{P}\left(Q^{*}, R^{*}\right)$, enstrophy production $\left\langle\omega_{i} S_{i j} \omega_{j} \mid Q^{*}, R^{*}\right\rangle \mathcal{P}\left(Q^{*}, R^{*}\right)$, and energy transfer $-\left\langle\operatorname{Tr}\left(\mathbf{A}^{2} \mathbf{A}^{T}\right) \mid Q, R\right\rangle \mathcal{P}\left(Q^{*}, R^{*}\right)$. Following Ref. 30, various quantities are normalized by the average transfer $\left|\left\langle\operatorname{Tr}\left(\mathbf{A}^{2} \mathbf{A}^{T}\right)\right\rangle\right|$.

\section{B. Enstrophy production, strain skewness, and energy transfer}

A similar study is performed with the various quantities entering in the definition of the third invariant $R$ [Eq. (20)], namely, the enstrophy production and the strain skewness. ${ }^{30}$ In Figs. 3(a)-3(d) these various quantities are shown, together with the predictions from the stochastic model. In all cases, it is apparent that model predictions are quite accurate. We see also that enstrophy production is clearly dominant in the $R^{*}<0$ and $Q^{*}>0$ quadrants. Let us mention that in the enstrophy-dissipation production dominated region $\left(R^{*}>0\right.$ and $Q^{*}>0$ ), enstrophy production becomes weakly negative, stating that in this region, enstrophy decreases with time [see Eq. (21)]. Also, in Figs. 3(a) and 3(b), we see that strain skewness is dominating in the bottom-right quadrant, but remains very important in the top-left quadrant. This is mainly linked to the fact that the evolution of dissipation not only depends on the strain skewness (or dissipation production) but also on enstrophy production and a term linked to the pressure Hessian [see Eq. (22)].

A related quantity of interest is

$$
-\operatorname{Tr}\left(\mathbf{A}^{2} \mathbf{A}^{\top}\right)=-\operatorname{Tr}\left(\mathbf{S}^{3}\right)-\frac{1}{4} \omega_{i} S_{i j} \omega_{j},
$$

which describes the time evolution of the pseudodissipation $d \operatorname{Tr}\left(\mathbf{A A}^{\top}\right) / d t=-\operatorname{Tr}\left(\mathbf{A}^{2} \mathbf{A}^{\top}\right)$ in the RE approximation. This 
quantity is sometimes called "energy transfer" $30,55,56$ when $\mathbf{A}$ is defined by filtering in the inertial range in the context of large eddy simulations (see Ref. 57). While here $\mathbf{A}$ is not filtered and therefore no such direct physical interpretation is available, this quantity is still presented as additional documentation of the properties of $\mathbf{A}$. Results are displayed in Figs. 3(e) and 3(f). Once again, the model reproduces well the trends observed in DNS, including negative regions in the top-right quadrant.

\section{Geometric alignments of vorticity with strain-rate eigenvectors}

An important universal feature of fully developed turbulent flows is the preferential alignment of vorticity along the eigendirection of the intermediate eigenvalue of the strainrate tensor $\mathbf{S}$ (see Ref. 7 and references therein). To study the alignment properties of vorticity conditioned on various values of $R$ and $Q$ the $(R, Q)$ plane is divided into four regions related to the eigenvalue structure of $\mathbf{A}$. Instead of the $Q$ $=0$ line to separate high and low rotation regions as was done in the qualitative discussions of the previous sections, we now use the quantitatively more precise classification, in which the $(R, Q)$ plane is divided into high and low rotation regions by the zero-discriminant line, i.e., $Q=-\left(27 R^{2} / 4\right)^{1 / 3}$.

Figure 4 shows the PDF of the cosine of the angle between vorticity and eigendirections with the most negative [Figs. 4(a) and 4(b)], intermediate [Figs. 4(c) and 4(d)], and most positive [Figs. 4(e) and 4(f)] eigenvalue of the stress, for both DNS and the model. The different symbols denote the results obtained in separate quadrants as separated by the Vieillefosse (i.e., zero discriminant) and the $R=0$ lines. In Figs. 4(g) and 4(h) is displayed the unconditional PDF independent of the quadrant, i.e., as obtained in all regions. As already observed in Ref. 34 the model predicts accurately the preferential alignment with the intermediate eigendirection (solid line), a trend of being orthogonal to the most contracting direction (dashed line), and an almost entirely decorrelated trend with the most extensive eigendirection (dashdotted line). The agreement between DNS and the model is excellent in all cases, even when conditioning on the separate quadrants. It is interesting to note that in Figs. 4(a) and 4(b) as well as in Figs. 4(e) and 4(f), the alignment PDF is essentially the same in three quadrants but very different in the $R>0$ and $Q>-\left(27 R^{2} / 4\right)^{1 / 3}$ quadrant. In Figs. 4(a) and 4(b) we observe that while the vorticity is mostly perpendicular to the most contracting eigendirection, in the topright quadrant the vorticity is, in fact, not orthogonal to the contracting eigendirection. This is the "vortex contracting" quadrant with an unstable focus and one contracting direction. This would suggest that the vorticity is aligned with the contracting direction. There is instead no strong preferred alignment but there is an almost zero probability that the vorticity is perpendicular to the contracting eigendirection. But, on average, when taking into account all the possible values for $R$ and $Q$ [Figs. 4(g) and 4(h)], vorticity remains weakly orthogonal to this eigendirection. In terms of the alignments with the intermediate eigendirection, one may have expected the preferential alignments to come mainly
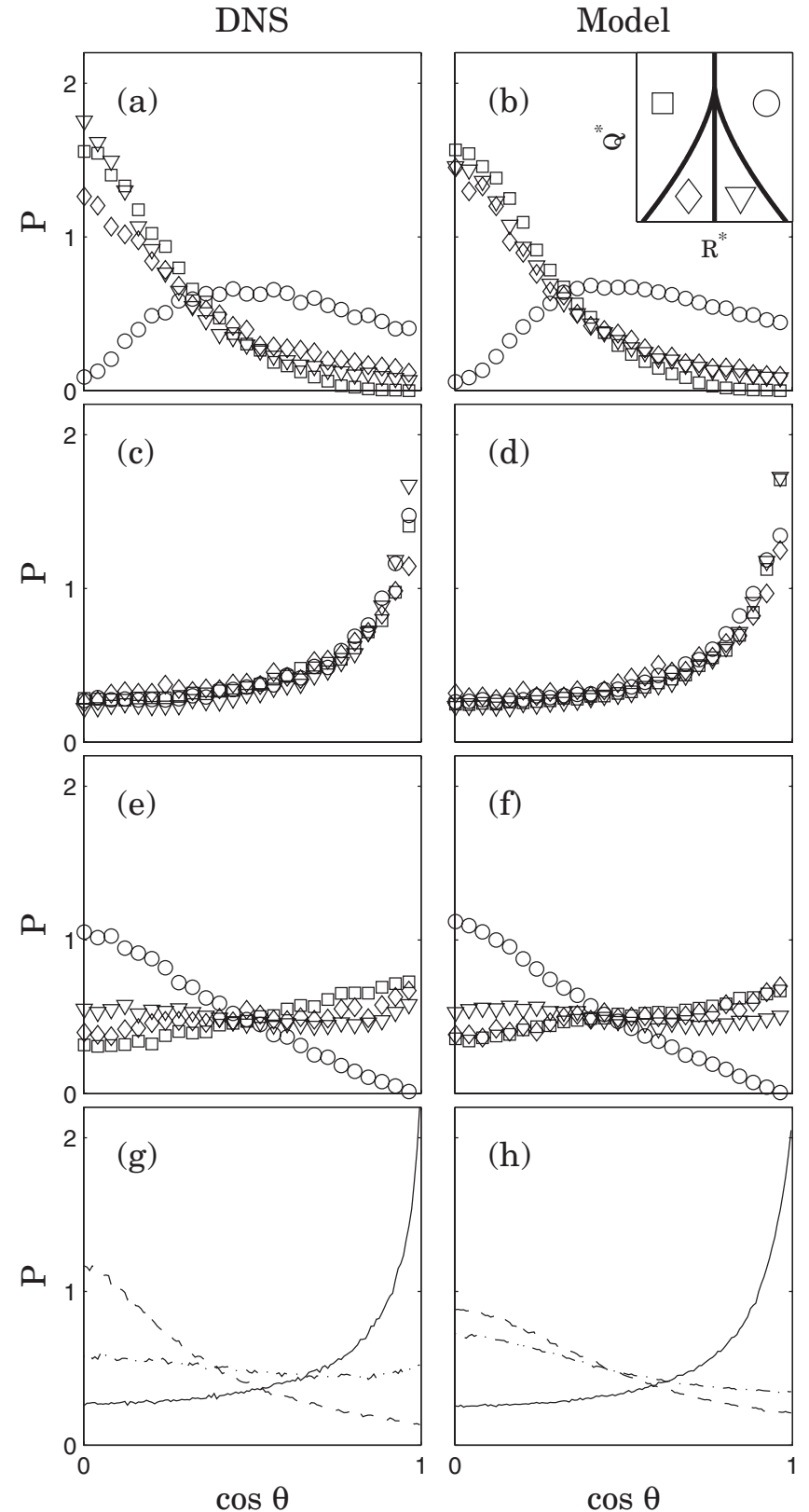

FIG. 4. PDF of the cosine of the angle $\theta$ between vorticity and the different eigendirections of $\mathbf{S}$ : [(a) and (b)] for the most contractive eigendirection (negative eigenvalue), [(c) and (d)] for the intermediate eigendirection, and [(e) and (f)] for the most positive (extensive) eigendirection. As it is schematically displayed in the inset of (b), different symbols are obtained from the four different regions of the $(R, Q)$ plane delimited by the $R>0$ and the Vieillefosse (or zero-discriminant) lines given by $Q=-\left(\frac{27}{4} R^{2}\right)^{1 / 3}:(\bigcirc) R>0$ and $Q>-\left(\frac{27}{4} R^{2}\right)^{1 / 3} ; \quad(\nabla) \quad R>0$ and $Q<-\left(\frac{27}{4} R^{2}\right)^{1 / 3} ; \quad(\square) \quad R<0$ and $Q>-\left(\frac{27}{4} R^{2}\right)^{1 / 3} ;(\diamond) R<0$ and $Q<-\left(\frac{27}{4} R^{2}\right)^{1 / 3}$. (g) and (h) show the unconditional PDF over the entire $(R, Q)$ plane. Different lines correspond to different associated eigenvalues: most negative (dashed), intermediate (solid), and most positive (dash-dotted).

from the bottom-right quadrant as predicted by the asymptotic diverging state of the RE equations. ${ }^{10,11}$ Nevertheless, in Figs. 4(c) and 4(d) we observe instead that the alignment with the intermediate eigendirection occurs quite independently of the characteristic values in the $(R, Q)$ plane. In Figs. 4(e) and 4(f) we observe that the alignment with the most extensive strain-rate eigendirection is random in all 
quadrants except, again, in the top-right quadrant where $R$ $>0$ and $Q>-\left(27 R^{2} / 4\right)^{1 / 3}$. Vortex "contraction," when it happens, appears to occur because it is mostly orthogonal to the extensive direction and also "not orthogonal" to the contracting direction, rather than being preferentially aligned with the contracting direction. The stochastic model predicts these nontrivial statistical geometric behaviors quite well.

\section{PRESSURE HESSIAN AND VISCOUS TERM}

Let us now focus directly on the terms requiring closure, namely, the pressure Hessian and the viscous term, instead of the statistics of the velocity gradient tensor considered in the previous section. One option could be to compare individual realizations of the model terms with the corresponding DNS values along Lagrangian trajectories. However, since these terms fluctuate greatly in the DNS, a statistically more robust comparison is performed using conditional averages, conditioned on $R$ and $Q$.

\section{A. Probability current and conditional averages}

The approach used in Ref. 16 is followed, based on a Fokker-Planck equation for the dynamics of $R$ and $Q$. To summarize the approach, we notice that along a Lagrangian trajectory, appropriately contracting (1) with $\mathbf{A}$ and $\mathbf{A}^{2}$, using the Cayley-Hamilton theorem, ${ }^{11}$ one can show that the time evolutions of the invariants $R^{*}$ and $Q^{*}$ are given by

$$
\frac{d Q^{*}}{d t^{*}}=-3 R^{*}-\frac{1}{\sigma^{3}} A_{i k} H_{k i}^{p}-\frac{1}{\sigma^{3}} A_{i k} H_{k i}^{\nu}
$$

and

$$
\frac{d R^{*}}{d t^{*}}=\frac{2}{3}\left(Q^{*}\right)^{2}-\frac{1}{\sigma^{4}} A_{i k} A_{k l} H_{l i}^{p}-\frac{1}{\sigma^{4}} A_{i k} A_{k l} H_{l i}^{\nu},
$$

where $\sigma^{2}=\left\langle S_{i j} S_{i j}\right\rangle$ is the strain variance and $t^{*}=\sigma t$ the nondimensional time. Also, $\mathbf{H}^{p}$ stands for (minus) the deviatoric part of the pressure Hessian, i.e.,

$$
H_{i j}^{p}=-\left(\frac{\partial^{2} p}{\partial x_{i} \partial x_{j}}-\frac{\delta_{i j}}{3} \frac{\partial^{2} p}{\partial x_{k} \partial x_{k}}\right),
$$

and $\mathbf{H}^{\nu}=\nu \nabla^{2} \mathbf{A}$ is the viscous term (recall that in the RE approximation, $\mathbf{H}^{p}=\mathbf{H}^{\nu}=0$ ). The Fokker-Planck equation describing the time evolution of the joint density $\mathcal{P}\left(Q^{*}, R^{*}\right)$ may be written as ${ }^{58}$

$$
\frac{\partial \mathcal{P}}{\partial t^{*}}+\left(\begin{array}{c}
\frac{\partial}{\partial Q^{*}} \\
\frac{\partial}{\partial R^{*}}
\end{array}\right) \cdot \mathcal{W}=0
$$

where the divergence of the probability current $\mathcal{W}$ controls time variations in the joint probability density $\mathcal{P}$. The probability current can be written in terms of conditional averages as

$$
\mathcal{W}=\left\langle\left(\begin{array}{l}
\frac{d Q^{*}}{d t^{*}} \\
\frac{d R^{*}}{d t^{*}}
\end{array}\right) Q^{*}, R^{*}\right) \mathcal{P}\left(Q^{*}, R^{*}\right) .
$$

It can be decomposed into $\mathcal{W}=\mathcal{W}_{R E}+\mathcal{W}_{p}+\mathcal{W}_{\nu}$, with

$$
\mathcal{W}_{R E}=\left\langle\left(\begin{array}{c}
-3 R^{*} \\
\frac{2}{3}\left(Q^{*}\right)^{2}
\end{array}\right) \mid Q^{*}, R^{*}\right\rangle \mathcal{P}\left(Q^{*}, R^{*}\right),
$$

which describes the deterministic (closed) part of the evolution of the two invariants,

$$
\mathcal{W}_{p}=\left\langle\left(\begin{array}{c}
-A_{i k} H_{k i}^{p} / \sigma^{3} \\
-A_{i k} A_{k l} H_{l i}^{p} / \sigma^{4}
\end{array}\right) \mid Q^{*}, R^{*}\right\rangle \mathcal{P}\left(Q^{*}, R^{*}\right),
$$

describing the pressure Hessian effects on the evolution of $R^{*}$ and $Q^{*}$ and, finally,

$$
\mathcal{W}_{\nu}=\left\langle\left(\begin{array}{c}
-A_{i k} H_{k i}^{\nu} / \sigma^{3} \\
-A_{i k} A_{k l} H_{l i}^{\nu} / \sigma^{4}
\end{array}\right) \mid Q^{*}, R^{*}\right\rangle \mathcal{P}\left(Q^{*}, R^{*}\right),
$$

describing the effects of the viscous term. An additional current might be considered in this description, linked to an additional forcing term that has been neglected in the Navier-Stokes equations [Eq. (1)]. This forcing is indeed negligible in front of the other terms of the right hand side of Eq. (1) since it can be written as the (small-scale) gradient of the large-scale forcing of the velocity, and thus, we will neglect its associated probability current.

Conversely, in the Fokker-Planck equation [Eq. (27)] for the joint probability distribution of $R^{*}$ and $Q^{*}$ obtained from the model [Eq. (18)], one has to take into account another term which comes from the delta-correlated Gaussian forcing. Appendix B provides the required background needed to compute the probability flux resulting from the stochastic forcing term in our model, i.e., the diffusion terms entering in Eqs. (24) and (25). It is shown that the currents associated with the deterministic and random parts of the joint stochastic evolution of $R$ and $Q$ predicted by the model [see Eq. (B4)] are of the same order of magnitude.

\section{B. Results}

In Fig. 5 the vector plots and associated streamlines corresponding to the various probability flux terms are presented. Both results obtained from DNS and from the model are shown.

First, as reference we present in Figs. 5(a) and 5(b) the closed RE current $\mathcal{W}_{\text {RE }}$ [Eq. (29)]. As is well known, ${ }^{10,11}$ the deterministic $\mathcal{W}_{\mathrm{RE}}$ probability current pushes probabilities toward the right tail of the Vieillefosse line. Since the model predicts accurately the joint probability $\mathcal{P}\left(Q^{*}, R^{*}\right)$, agreement between DNS and the model predictions (length of vectors) is quite good because the self-stretching term $-\mathbf{A}^{2}$ is taken into account exactly in the model [Eq. (18)].

The action of the pressure Hessian, given by the probability current $\mathcal{W}_{p}$ and shown in Figs. 5(c) and 5(d), is quite interesting. From the DNS data, two main pressure Hessian effects can be observed. First, the pressure Hessian counteracts the effects induced by the RE terms since the flux goes 


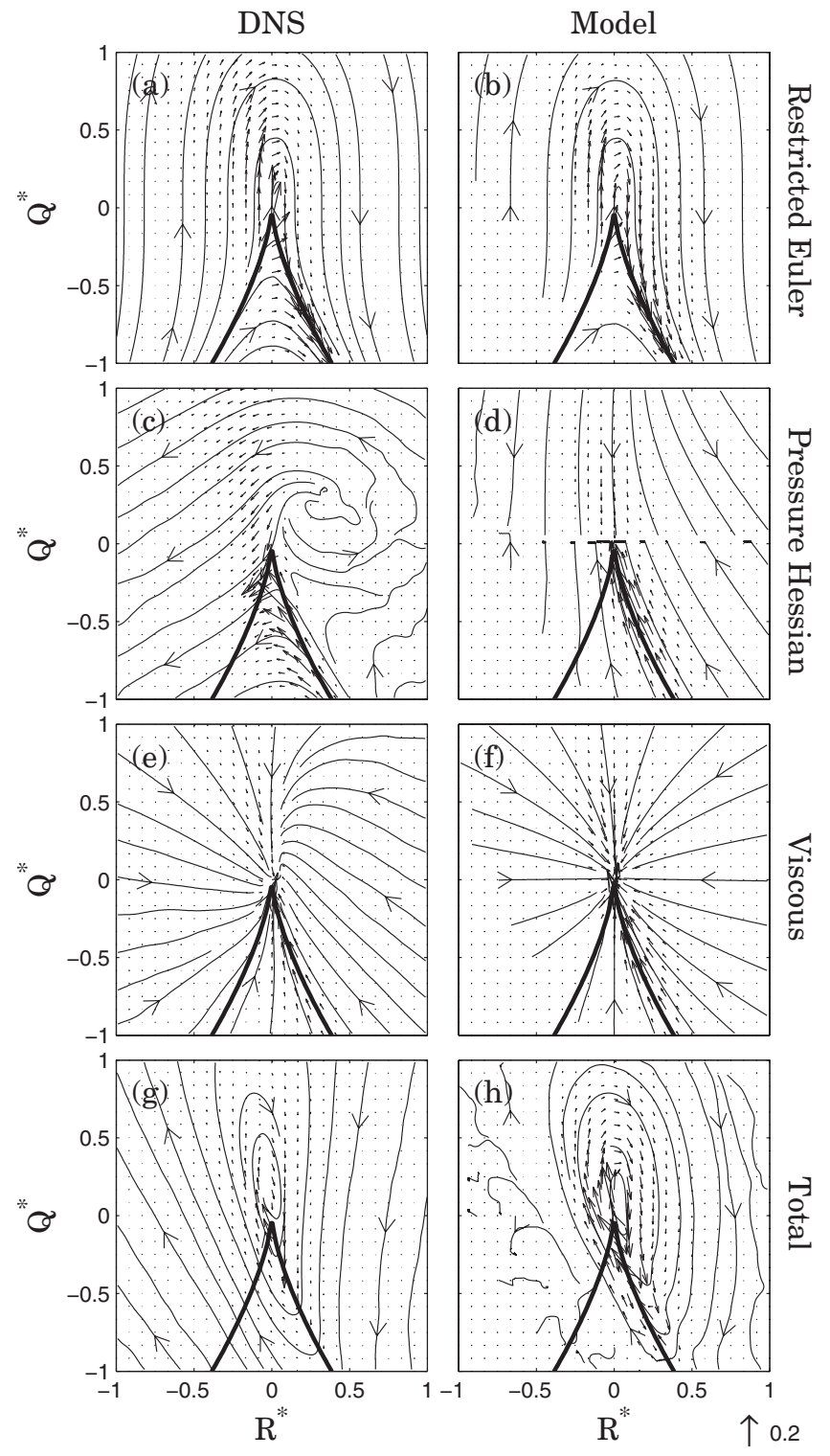

FIG. 5. Vector and streamline plots of the probability current associated with the [(a) and (b)] RE approximation [Eq. (29)], [(c) and (d)] the pressure Hessian [Eq. (30)], and [(e) and (f)] the viscous term [Eq. (31)]. The total current [Eq. (28)] is represented in (g) and (h). The scale of the vectors is the same in all plots and a reference is given below $(\mathrm{h})$, whose (nondimensional) magnitude is $2 \times 10^{-1}$.

toward the center of the $R Q$ plane along the right tail Vieillefosse line. This feature is well reproduced by the model, with vector magnitudes of the same order. Another important effect of the pressure Hessian is that in the $R<0$ left half-plane, the probability current leads the probability toward the left tail of the Vieillefosse line, namely, toward the dissipation-enstrophy production dominated region (in lower-left direction). This feature is not reproduced by the model, which instead appears to act exclusively in the vertical direction, upward in the $Q<0$ plane, and downward in the $Q>0$ side. This explains perhaps why the model leads to an underestimation (see Fig. 1) of the probability of dissipation-enstrophy production events (i.e., the bottom-left quadrant). At the same time, the absence of "leftward" flux out of the enstrophy-dissipation production region (i.e., top-

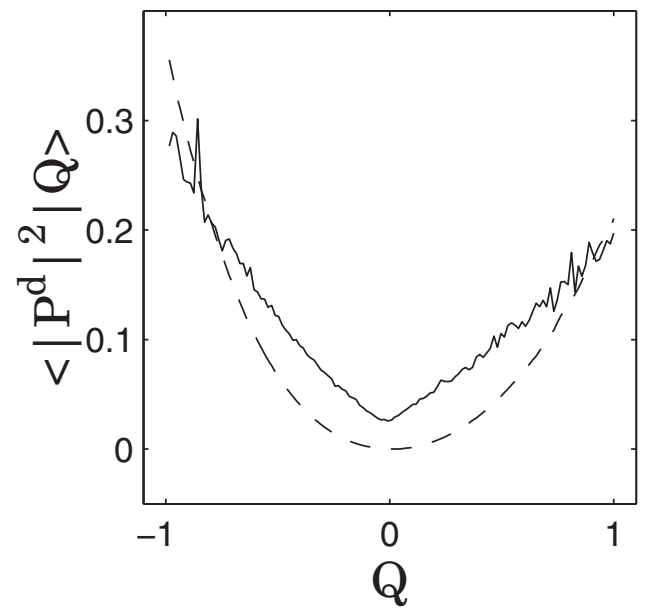

FIG. 6. Conditional average of the norm square of the deviatoric part of the pressure Hessian $\left\langle\left|\mathbf{H}^{p}\right|^{2} \mid Q\right\rangle$ with respect to the invariant $Q$. Both $\left|\mathbf{H}^{p}\right|^{2}$ and $Q$ are nondimensionalized by their respective standard deviations. DNS results (solid line) and model predictions (dashed line) are shown.

right quadrant) may explain why the model overpredicts the probability of events in that quadrant. A very marked feature of the DNS results is that the magnitudes of the vectors are essentially negligible in the entire vortex contraction quadrant above the right Vieillefosse line $[R>0$ and $\left.Q>-\left(27 R^{2} / 4\right)^{1 / 3}\right]$, leading to some uncertainty in the computed streamlines there.

Another main difference between DNS and model predictions is the fact that for the model, $\mathcal{W}_{p}$ vanishes at vanishing $Q$, but not for the DNS. A general feature of the pressure Hessian model is that its deviatoric part is directly proportional to $\operatorname{Tr}\left(\mathbf{A}^{2}\right)=-2 Q$ [see Eq. (16)]. Incidentally, the same occurs in further generalizations that have been proposed by Gibbon and Holm ${ }^{20}$ [see their Eq. (5.8)], namely,

$$
\boldsymbol{\nabla} \boldsymbol{\nabla} p=-\left[\sum_{n=1}^{N} c_{n} \frac{\mathbf{G}_{\mathbf{n}}}{\operatorname{Tr}\left(\mathbf{G}_{\mathbf{n}}\right)}\right] \operatorname{Tr}\left(\mathbf{A}^{2}\right) \quad \text { with } \sum_{n=1}^{N} c_{n}=1,
$$

where the scalars $c_{N}$ are undetermined and $\mathbf{G}_{\mathbf{n}}$ are any nonsingular symmetric tensors. Once again, we can see that the deviatoric part of the pressure Hessian $\mathbf{H}^{p}$ is still proportional to $\operatorname{Tr}\left(\mathbf{A}^{2}\right)$. For the sake of completeness, let us remark that at least formally, this issue does not arise in the matrix shell model of Ref. 36. This is because the nonlocal closure terms in the matrix shell model ${ }^{36}$ are not directly proportional to $\operatorname{Tr}\left(\mathbf{A}^{2}\right)$ since the connection to pressure Hessian and Poisson equation for pressure is not included in that approach. It would be very interesting to check if the comparison with DNS for the equivalent probability current in the matrix shell model, i.e., based on relevant portions of the quadratic nonlinear interaction terms, is better or not. Such studies are left for future work.

To more clearly isolate the behavior of the pressure Hessian near the $Q=0$ line, we study the magnitude of the anisotropic (i.e., deviatoric) part of the pressure Hessian, conditioned on the local value of $Q$. Figure 6 shows the conditional average of the norm (square) of the deviatoric part of the pressure Hessian, i.e., $\left\langle\left|\mathbf{H}^{p}\right|^{2} \mid Q\right\rangle$, where $\left|\mathbf{H}^{p}\right|^{2}$ $=\operatorname{Tr}\left[\mathbf{H}^{p}\left(\mathbf{H}^{p}\right)^{\top}\right]$, as a function of the local value of the invari- 
ant $Q$, for both the DNS and the model. For vanishing $Q$, the conditional average $\left|\mathbf{H}^{p}\right|^{2}$ from the DNS does not vanish. As discussed before this property is not reproduced by the existing models for the pressure Hessian, namely, the tetrad model [Eq. (12)], the CM06 model [Eq. (16)], and the generalized tetrad model [Eq. (32)], because all of them predict a pressure Hessian proportional to $Q$. Second, one can see that for the range of $Q$ under consideration (i.e., $Q$ $\left.\in\left[-\sigma_{Q}, \sigma_{Q}\right]\right)\left(\sigma_{Q}\right.$ stands for the standard deviation of $\left.Q\right)$, the conditional average of $\left|\mathbf{H}^{p}\right|^{2}$ behaves as $\sim|Q|$ (up to a positive additive constant), whereas the model predicts it to be proportional to $Q^{2}$. The fact that the model predicts a quadratic behavior can be understood from Taylor's development, namely, $\mathbf{H}^{p} \approx-Q \tau_{K} \mathbf{S}$, leading to $\left\langle\left|\mathbf{H}^{p}\right|^{2} \mid Q\right\rangle \sim Q^{2}$ since $\left\langle\tau_{K}^{2}|\mathbf{S}|^{2}\right\rangle \sim 1$. The small asymmetry in the quadratic behavior seen in Fig. 6 is caused by higher order terms entering in the expansion for the model.

For the viscous term, one observes in Figs. 5(e) and 5(f) that the model reproduces the probability flux reasonably well. Consistent with the observations already made in Ref. 16 , the viscous effect is to push the probabilities toward vanishing $R$ and $Q$, not only along the Vieillefosse line but also everywhere else. We notice that the model overpredicts the magnitudes, i.e., at this Reynolds number the model provides too strong damping but is qualitatively correct.

In Figs. 5(g) and 5(h) is shown the sum of all these terms, namely, the total probability current $\mathcal{W}=\mathcal{W}_{\mathrm{RE}}$ $+\mathcal{W}_{p}+\mathcal{W}_{\nu}$. For the model case, another term coming from the Gaussian delta-correlated forcing [see Appendix B and Eq. (B9)] has been added. The circular motion around the origin of the $R Q$ plane has already been reported in Refs. 15 and 30. At this point, from Fig. 5 one can observe that all the terms (self-stretching, pressure Hessian, and viscous Laplacian) entering in the Navier-Stokes equations [Eq. (1)] are of the same order of magnitude (viscous Laplacian is a little bit smaller than the two other terms, but not by much). Similar conclusions can be drawn for the deterministic terms entering in the model [Eq. (18) and Figs. 5(b)-5(f)], although, as shown in Appendix B, the amplitude of the forcing is not negligible either. Focusing on the total probability current [Figs. 5(g) and 5(h)], we reach the conclusion that the fact that the modeled pressure Hessian [Fig. 5(d)] is not able to reproduce the probability flux toward $R^{*}<0$ and $Q^{*}<0$ regions as it is observed in DNS [Fig. 5(c)], explains why the model overpredicts $R^{*}>0$ and $Q^{*}>0$ regions and underpredicts $R^{*}<0$ and $Q^{*}<0$ regions, as observed in Fig. 1. We also remark that streamlines shown in the left part of Fig. 5 (h) are not significant because of the very low values of the joint probability $\mathcal{P}\left(Q^{*}, R^{*}\right)$.

\section{VORTICITY ALIGNMENTS WITH PRESSURE HESSIAN AND VISCOUS LAPLACIAN EIGENDIRECTIONS}

\section{A. Pressure Hessian}

Here we focus on vorticity alignment properties along the eigendirections of the pressure Hessian. It has been derived, in the inviscid limit (Euler equations) $)^{50,59,60}$ that vor- ticity $\omega_{i}$ tends to be simultaneously an eigenvector of the rate-of-strain tensor $\mathbf{S}$ and the pressure Hessian. When $\nu=0$ [see Eq. (21)],

$$
\frac{d \omega_{i}}{d t}=S_{i j} \omega_{j}
$$

and taking another time derivative and using the time evolution of $\mathbf{S}$ [see Eq. (22)], we get

$$
\frac{d^{2} \omega_{i}}{d t^{2}}=-\frac{\partial^{2} p}{\partial x_{i} \partial x_{j}} \omega_{j}
$$

Following Ref. 50, we then notice that if vorticity of a fluid particle continues to be an eigenvector of the rate-of-strain tensor, then it is also an eigenvector of the pressure Hessian. To see if such a trend is observed in a finite viscosity turbulent flow we will quantify the alignments of vorticity with the eigendirections of the pressure Hessian. Such an analysis based on DNS has been already performed, ${ }^{61,62}$ but here the purpose is to compare results with the predictions of the model.

Let us focus on alignment properties of vorticity with respect to the eigendirections of the deviatoric part of the pressure Hessian [i.e., $-H_{i j}^{p}$ defined in Eq. (26)]. Alignment PDFs are shown in Fig. 7, presented in a similar fashion as in Fig. 4. We can see in Fig. 7(a) that vorticity is preferentially orthogonal to the eigendirection of the smallest eigenvalue, except in the top-left quadrant [i.e., $R<0$ and $\left.Q>-\left(27 / 4 R^{2}\right)^{1 / 3}\right]$ where the local topology is dominated by one direction of stretching and a stable focus. Also in this quadrant, vorticity is preferentially aligned with the extending eigendirection. The model predicts a slightly different picture since vorticity is predicted to be also preferentially orthogonal to the eigendirection except in the top-right quadrant [i.e., $R>0$ and $Q>-\left(27 / 4 R^{2}\right)^{1 / 3}$ ] for which local topology is dominated by one compressive direction and an unstable focus.

In Figs. 7(c) and 7(d), we focus on the pressure Hessian eigendirection of its intermediate eigenvalue. In a similar way as with eigendirections of the rate of strain, vorticity is preferentially aligned with this eigendirection in all the quadrants, and this is also very well predicted by the model. About the eigendirection of the largest eigenvalue, we can see that for the DNS [Fig. 7(e)] vorticity is weakly preferentially aligned with the eigendirection, except again in the top-left quadrant where vorticity is clearly preferentially orthogonal to this eigendirection. In Fig. 7(f), we can see that the model predicts most of the trends quite well in all the quadrants. For the average results over all quadrants, it can be seen in Figs. 7(g) and 7(h) that the model predicts with a fairly good accuracy the behavior of vorticity with the eigendirection of the intermediate eigenvalue (although it overpredicts the peak a little bit). In the other extremal eigendirections, the model reproduces the moderate peak at $\cos (\theta)$ $\sim 0$ but misses the narrow peaks near alignment at $\cos (\theta) \sim 1$.

The fact that the model reproduces very well the events for which vorticity happens to be an eigenvector of the rateof-strain tensor can be understood phenomenologically in the 


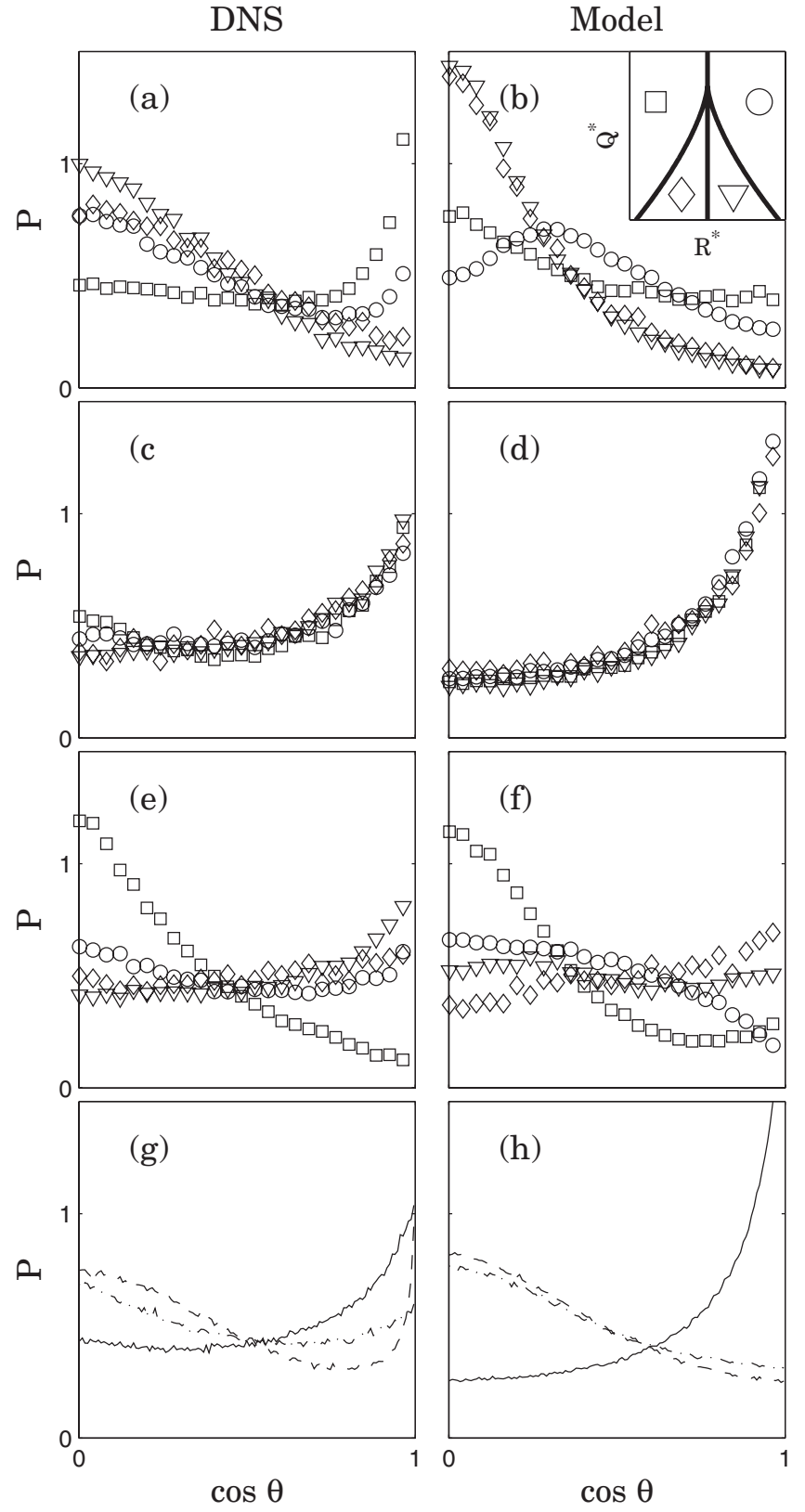

FIG. 7. PDFs of the cosine of the angle between vorticity and the different eigendirections of pressure Hessian tensor $\mathbf{H}^{p}$ : [(a) and (b)] for the smallest eigenvalue eigendirection, [(c) and (d)] for the intermediate eigendirection, and [(e) and (f)] for the most positive eigendirection. As it is schematically displayed in the inset of (b), in a similar fashion as in Fig. 4, different symbols are obtained from the four different regions of the $(R, Q)$ plane delimited by the $R>0$ and the Vieillefosse (or zero-discriminant) lines given by $Q=-\left(\frac{27}{4} R^{2}\right)^{1 / 3}: \quad(\bigcirc) \quad R>0$ and $Q>-\left(\frac{27}{4} R^{2}\right)^{1 / 3} ; \quad(\nabla) \quad R>0$ and $Q<-\left(\frac{27}{4} R^{2}\right)^{1 / 3} ; \quad(\square) \quad R<0 \quad$ and $Q>-\left(\frac{27}{4} R^{2}\right)^{1 / 3} ; \quad(\diamond) \quad R<0 \quad$ and $Q<-\left(\frac{27}{4} R^{2}\right)^{1 / 3}$. (g) and (h) show the unconditional PDF over the entire $(R, Q)$ plane. Different lines correspond to different associated eigenvalues: most negative (dashed), intermediate (solid), and most positive (dashdotted).

following way. When vorticity is an eigenvector of the rate of strain, i.e., $\mathbf{S} \boldsymbol{\omega}=\beta \boldsymbol{\omega}$, then vorticity is also an eigenvector of the velocity gradient tensor itself, namely, $\mathbf{A} \boldsymbol{\omega}=(\mathbf{S}$ $+\boldsymbol{\Omega}) \boldsymbol{\omega}=\beta \boldsymbol{\omega}$, since by definition $\boldsymbol{\Omega} \boldsymbol{\omega}=0$. Let us notice that vorticity is also an eigenvector of $\mathbf{A}^{\top}=\mathbf{S}-\boldsymbol{\Omega}$ with the same eigenvalue $\beta$. It is then straightforward to show by induction that for any power $n \in \mathbb{N}, \mathbf{A}^{n} \boldsymbol{\omega}=\left(\mathbf{A}^{\top}\right)^{n} \boldsymbol{\omega}=\beta^{n} \boldsymbol{\omega}$. For these
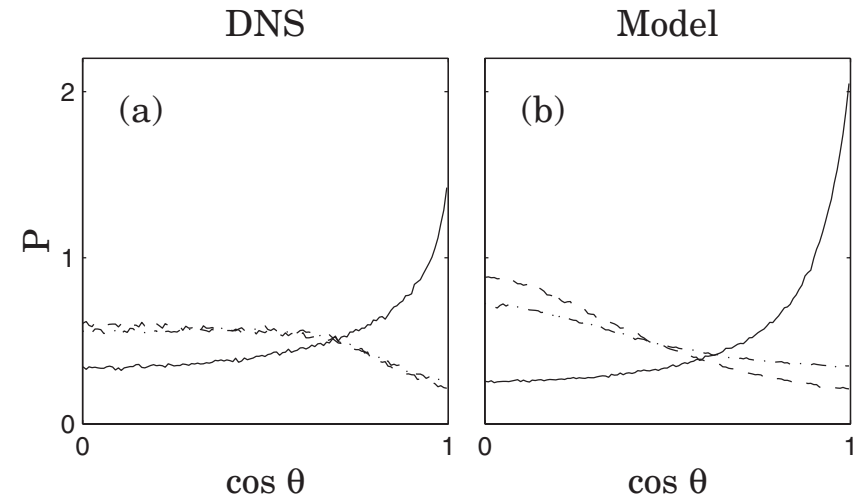

FIG. 8. PDF of the cosine between vorticity and eigendirections of $\nu \Delta \mathbf{S}$ associated with the smallest (dashed line), intermediate (solid line), and biggest (dot-dashed line) eigenvalues of the strain-rate tensor.

very particular events in which vorticity is an eigenvector of the rate of strain, one notices that the matrix exponential entering the (inverse) recent Cauchy-Green tensor [in Eq. (15)] can be written as

$$
\mathbf{C}_{\tau_{K}}^{-1}=e^{-\tau_{K} \mathbf{A}^{\top}} e^{-\tau_{K} \mathbf{A}}=\sum_{n, m=0}^{+\infty} \frac{\left(-\tau_{K}\right)^{n+m}}{n ! m !}\left(\mathbf{A}^{\top}\right)^{n} \mathbf{A}^{m} .
$$

From Eq. (35) it is easily seen that vorticity is also an eigenvector of both the recent Cauchy-Green tensor [Eq. (15)] and its inverse. For example, $\mathbf{C}_{\tau_{K}}^{-1} \boldsymbol{\omega}=e^{-2 \tau_{K} \beta} \boldsymbol{\omega}$. Finally, since the pressure Hessian is modeled as proportional to $\mathbf{C}_{\tau_{K}}^{-1}$ [Eq. (16)], we can state here that the present model is such that when the vorticity is an eigenvector of the rate of strain, then it is also an eigenvector of the modeled pressure Hessian, the ordering of the associated eigenvalues being respected in absolute value. More precisely, if vorticity is an eigenvector of $\mathbf{S}$ with respective eigenvalue $\beta$, then vorticity is also an eigenvector of the pressure Hessian with eigenvalue $-\left[\operatorname{Tr}\left(\mathbf{A}^{2}\right) / \operatorname{Tr}\left(\mathbf{C}_{\tau_{K}}^{-1}\right)\right] e^{-2 \tau_{K} \beta}$.

\section{B. Viscous term}

Let us now focus on the geometrical properties of the viscous tensor, namely, $\nu \nabla^{2} \mathbf{A}$, appearing in Eq. (1). Let us begin with its symmetric part $\nu \nabla^{2} \mathbf{S}$. The present model [i.e., Eq. (18)] contains the closure for the viscous term as written in Eq. (14) that stated that the Laplacian of $\mathbf{A}$ is proportional to $\mathbf{A}$ itself. In terms of eigendirections, it is assumed that both $\mathbf{S}$ and $\nabla^{2} \mathbf{S}$ have the same eigendirections. Among others, alignment properties of vorticity $\boldsymbol{\omega}$ and eigendirections of $\nabla^{2} \mathbf{S}$ should be exactly the same as alignments of vorticity with eigendirections of $\mathbf{S}$. To determine whether this is observed in DNS flows, we present in Fig. 8 the PDFs of the cosine of the angle between vorticity and eigendirections of the viscous term, for both DNS [Fig. 8(a)] and the model [Fig. 8(b)]. Figure 8(b) is, in fact, the same as Fig. 4(h) and is reproduced here for convenience. We see clearly that the overall geometrical picture is really close between DNS and the model, and that there is preferential alignment of vorticity with the eigendirection associated with the intermediate eigenvalue of the Laplacian term as well. 


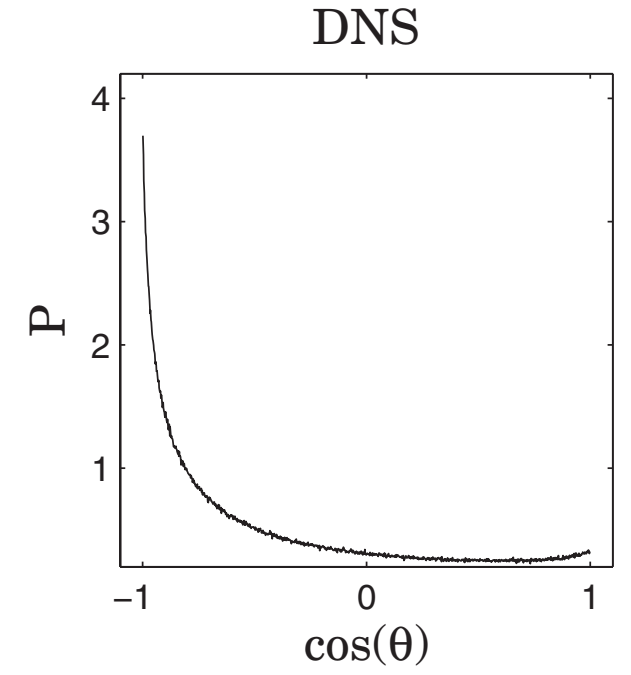

FIG. 9. PDF of the cosine of the angle $\theta$ between vorticity $\omega_{i}=-\frac{1}{2} \varepsilon_{i j k} \Omega_{j k}$ and $\varpi$, the Laplacian of vorticity vector $\varpi_{i}=-\frac{1}{2} \varepsilon_{i j k} \nu \nabla^{2} \Omega_{j k}$, obtained from DNS.

Let us now focus on the antisymmetric part of the viscous term, namely, $\nu \nabla^{2} \boldsymbol{\Omega}$. The vorticity vector is given by $\omega_{i}=-\frac{1}{2} \varepsilon_{i j k} \Omega_{j k}$. The viscous term $\varpi=\nu \nabla^{2} \boldsymbol{\omega}$ can also be written as $\varpi_{i}=-\frac{1}{2} \varepsilon_{i j k} \nu \nabla^{2} \Omega_{j k}$. The model for the viscous term [Eq. (17)] implies that the angle $\theta=(\varpi, \boldsymbol{\omega})$ between the vorticity and the vorticity Laplacian is fixed and equals $\pi$, i.e., same direction but opposite orientation, since the model [Eq. (17)] is proportional to the velocity gradient tensor $\mathbf{A}$ with a negative coefficient. One may wonder if this is consistent with DNS data. We represent in Fig. 9 the PDF of $\cos \theta$ estimated from the same DNS fields. Clearly we see that the two vectors share preferentially the same direction, but opposite orientation. The model is therefore consistent with the observed alignment trends of the full Laplacian of velocity gradient.

\section{CONCLUSIONS}

Extensive comparisons have been made between predictions of a new stochastic Lagrangian model for the velocity gradient tensor and results from DNS at a corresponding moderate Reynolds number. The model reproduces many inherent geometric and statistical properties of small-scale turbulence quite well. The statistics of alignment angles between vorticity and the principal axes of the rate-of-strain tensor are very well reproduced. The joint statistics of velocity gradient invariants $R$ and $Q$ are also reproduced well. Specifically, the joint PDF's elongation into the top-left and bottom-right quadrants observed in the DNS also occurs in the model. Some differences occur in the model in the topright and bottom-left quadrants. In order to directly assess the action of the modeled pressure and viscous terms in a statistically robust fashion that takes into account the local topology of the flow, the probability current $\mathcal{W}$ has been studied. The agreement between DNS and model predictions is good near the dominant Vieillefosse tail in the lower-right quadrant of the $(R, Q)$ plane. However, in the dissipationenstrophy production dominated region (bottom left), the model does not reproduce the true dynamics and requires further developments. Finally, the alignment properties of vorticity with respect to the principal axes of the pressure Hessian tensor have been studied. The model reproduces quite well the preferential alignment of vorticity with the eigendirection associated with the intermediate eigenvalue. We elucidate the fact that in the model an eigenvector of the rate of strain is also an eigenvector of the pressure Hessian, and this is, in fact, consistent with known behavior of vorticity in the inviscid limit (i.e., the Euler equations).

This analysis has confirmed that the stochastic model is capable of predicting many nontrivial properties of smallscale turbulence as described by the geometric and statistical properties of the velocity gradient tensor. Nevertheless, there appear to be difficulties in specific regions of the flow, especially those in which the vorticity is being contracted such as in the top-right or bottom-left portion of the invariant $(R, Q)$ plane. Whether these drawbacks of the model are also related to the difficulties observed when raising the Reynolds number of the flow ${ }^{34,35}$ also remains to be explored.

\section{ACKNOWLEDGMENTS}

We thank S. Chen, R. Chétrite, G. Eyink, K. Gawedzki, and Y. Li for useful discussions. L.C. is supported by a postdoctoral fellowship from the Keck Foundation and C.M. by the National Science Foundation (Grant No. AST-0428325).

\section{APPENDIX A: DEFINITION AND IMPLEMENTATION OF TENSOR GAUSSIAN FORCING}

In this appendix, the tensorial Gaussian forcing $d \mathbf{W}$ entering in the model [Eq. (18)] is described. It can be written as

$$
d W_{i j}=D_{i j k l} d B_{k l},
$$

where $D_{i j k l}$ are the diffusion coefficients and $d \mathbf{B}$ is a tensorial isotropic Wiener process, whose components are such that

$$
\left\langle d B_{i j}\right\rangle=0 \quad \text { and } \quad\left\langle d B_{i j} d B_{k l}\right\rangle=2 d t \delta_{i k} \delta_{j l} .
$$

The coefficients $D_{i j k l}$ are chosen such that the noise $d \mathbf{W}$ is consistent with a trace-free, homogeneous, and isotropic tensor of a given unit variance, namely, $\left\langle d W_{i j} d W_{k l}\right\rangle$ $=2 d t D_{i j p q} D_{k l p q}$, with

$$
D_{i j p q} D_{k l p q}=2 \delta_{i k} \delta_{j l}-\frac{1}{2} \delta_{i j} \delta_{k l}-\frac{1}{2} \delta_{i l} \delta_{j k} .
$$

As a consequence, longitudinal components of the noise $d \mathbf{W}$ are of variances $2 d t$ and $4 d t$ for the transverse ones. Also, let us recall that the dimension of the diffusion coefficients is time $^{-3 / 2}$. If the tensor $\mathbf{D}$ is assumed isotropic itself, then the unique solution of Eq. (A3) is given by

$$
D_{i j p q}=a \delta_{i j} \delta_{p q}+b \delta_{i p} \delta_{j q}+c \delta_{i q} \delta_{j p},
$$

with

$$
a=\frac{1}{3} \frac{3+\sqrt{15}}{\sqrt{10}+\sqrt{6}}, \quad b=-\frac{\sqrt{10}+\sqrt{6}}{4}, \quad c=\frac{1}{\sqrt{10}+\sqrt{6}} .
$$




\section{APPENDIX B: SDES}

The basic tensorial SDE [Eq. (18)] can be written as

$$
d A_{i j}=V_{i j} d t+D_{i j k l} d B_{k l}
$$

where $V_{i j}$ are the drift coefficients representing the selfstretching, pressure Hessian, and viscous terms entering in Eq. (18) and $D_{i j k l} d B_{k l}$ is the forcing term described in Appendix A. The associated Fokker-Planck equation for the joint probability of the velocity gradients $f(\mathbf{A} ; t)$ is given by

$$
\frac{\partial f}{\partial t}=-\frac{\partial}{\partial A_{i j}}\left[f V_{i j}\right]+\frac{\partial^{2}}{\partial A_{i j} \partial A_{k l}}\left[f D_{i j p q} D_{k l p q}\right]
$$

Here we are interested in the joint probability $\mathcal{P}(Q, R)$ of two invariants of the velocity gradients, namely, $R$ and $Q[\mathrm{cf}$. Eq. (27)]. In Itô interpretation, both the stochastic equations governing the dynamics of $R$ and $Q$ and the associated Fokker-Planck equation can be computed (cf. Refs. 29, 52, 58, and 63) from the evolution of $\mathbf{A}$ [Eq. (B1)]. To do so, one needs to know how a SDE is written under a nonlinear transformation since $R$ and $Q$ are nonlinear functions of the components of A.

In general terms, let us call such a time dependent nonlinear transformation $\boldsymbol{\xi}(t, \mathbf{A}): \mathbf{A} \mapsto \boldsymbol{\xi}(t, \mathbf{A})$ with components $\xi_{k}, k \in\{1,2, \ldots, N\}$. Starting from the SDE of $\mathbf{A}[$ [Eq. (B1)], general formula ${ }^{29,52,58,63}$ gives the new SDEs that governs $\boldsymbol{\xi}$, namely,

$$
\begin{aligned}
d \boldsymbol{\xi}= & {\left[\frac{\partial \boldsymbol{\xi}}{\partial t}+\frac{\partial \boldsymbol{\xi}}{\partial A_{i j}} V_{i j}+\frac{1}{2} \frac{\partial^{2} \boldsymbol{\xi}}{\partial A_{i j} \partial A_{p q}} D_{i j r s} D_{p q r s}\right] d t } \\
& +\frac{\partial \boldsymbol{\xi}}{\partial A_{i j}} D_{i j r s} d B_{r s} .
\end{aligned}
$$

To compute the time evolution of the invariants $R$ and $Q$, we use Eq. (B3) with the particular (time independent) nonlinear transformations $\xi_{1}=Q=-\operatorname{Tr}\left(\mathbf{A}^{2}\right) / 2=-A_{i j} A_{j i} / 2$ and $\xi_{2}$ $=R=-\operatorname{Tr}\left(\mathbf{A}^{3}\right) / 3=-A_{i j} A_{j k} A_{k i} / 3$. We get

$$
\begin{aligned}
& d Q=\left[-V_{i j} A_{j i}-\frac{1}{2} D_{i j p q} D_{j i p q}\right] d t-A_{j i} D_{i j p q} d B_{p q}, \\
& d R=\left[-V_{i j} A_{j q} A_{q i}-A_{l i} D_{i j p q} D_{j l p q}\right] d t-A_{j r} A_{r i} D_{i j p q} d B_{p q},
\end{aligned}
$$

where, using former notations, we notice that $-V_{i j} A_{j i}=-3 R$ $-H_{i j}^{p} A_{j i}-H_{i j}^{\nu} A_{j i}$ and, using the Cayley-Hamilton theorem,
$-V_{i j} A_{j q} A_{q i}=\frac{2}{3} Q^{2}-H_{i j}^{p} A_{j q} A_{q i}-H_{i j}^{\nu} A_{j q} A_{q i} . \quad$ Furthermore, the "spurious" drift terms coming from the delta-correlated Gaussian noise vanish, i.e., using Eq. (A3) one can show that $D_{i j p q} D_{j i p q}=0$ and $A_{l i} D_{i j p q} D_{j l p q}=0$. In a straightforward manner, ${ }^{29,52,58,63}$ we get from Eq. (B4) the corresponding Fokker-Planck equation for the joint probability $\mathcal{P}(Q, R)$,

$$
\frac{\partial \mathcal{P}}{\partial t}=-\frac{\partial}{\partial \xi_{i}}\left[\mathcal{P} N_{i}\right]+\frac{\partial}{\partial \xi_{i} \partial \xi_{j}}\left[\mathcal{P} M_{i j}\right] .
$$

In Eq. (B5), the new coefficients $N_{i}$ and $M_{i j}$ can be easily obtained from Eq. (B4), although they are not known as functions of $\xi_{1}=Q$ and $\xi_{2}=R$. Therefore, we will use conditional averages to estimate them. Henceforth, we will write the Fokker-Planck equation with conditional averages and obtain

$$
\frac{\partial \mathcal{P}}{\partial t}=-\frac{\partial}{\partial \xi_{i}}\left\langle\mathcal{P} N_{i} \mid \xi\right\rangle+\frac{\partial}{\partial \xi_{i} \partial \xi_{j}}\left\langle\mathcal{P} M_{i j} \mid \xi\right\rangle,
$$

where the drift coefficients $N_{i}$ are given by

$$
N_{i}=\left(\begin{array}{c}
-3 R-H_{i j}^{p} A_{j i}-H_{i j}^{\nu} A_{j i} \\
\frac{2}{3} Q^{2}-H_{i j}^{p} A_{j q} A_{q i}-H_{i j}^{\nu} A_{j q} A_{q i}
\end{array}\right)
$$

and the diffusion elements by

$$
\begin{aligned}
& M_{11}=2 \operatorname{Tr}\left(\mathbf{A} \mathbf{A}^{\top}\right)+Q, \\
& M_{12}=M_{21}=2 \operatorname{Tr}\left(\mathbf{A}^{\top} \mathbf{A}^{2}\right)+\frac{3}{2} R, \\
& M_{22}=2 \operatorname{Tr}\left[\left(\mathbf{A}^{\top}\right)^{2} \mathbf{A}^{2}\right]-2 Q^{2}-\frac{1}{2} \operatorname{Tr}\left(\mathbf{A}^{4}\right) .
\end{aligned}
$$

Finally, using again the general transformation (B3), the probability current $\mathcal{W}$ of the joint probability $\mathcal{P}\left(Q^{*}, R^{*}\right)$ of the nondimensional invariants $Q^{*}$ and $R^{*}$, entering in the nondimensional Fokker-Planck equation

$$
\frac{\partial \mathcal{P}}{\partial t^{*}}+\left(\begin{array}{c}
\frac{\partial}{\partial Q^{*}} \\
\frac{\partial}{\partial R^{*}}
\end{array}\right) \cdot \mathcal{W}=0
$$

is given by $\mathcal{W}=\mathcal{W}_{\text {drift }}+\mathcal{W}_{\text {diff }}$ with (we recall that $\sigma^{2}$ $\left.=\left\langle S_{i j} S_{i j}\right\rangle\right)$

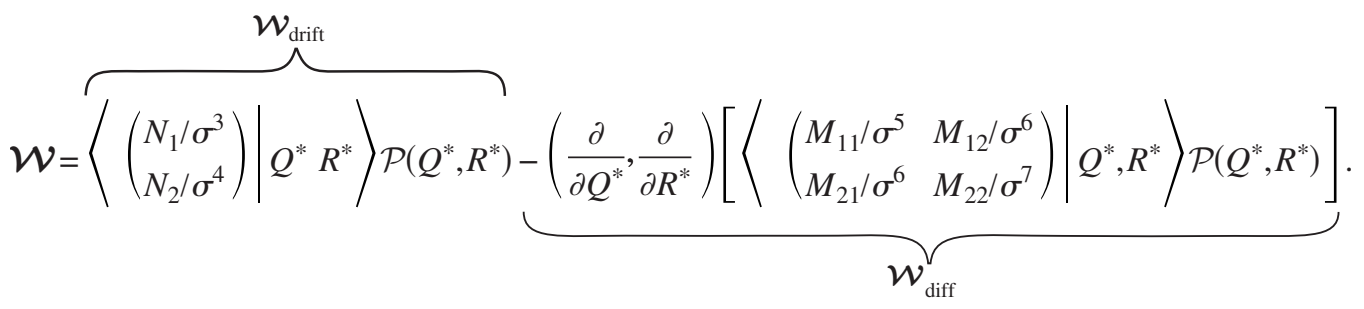



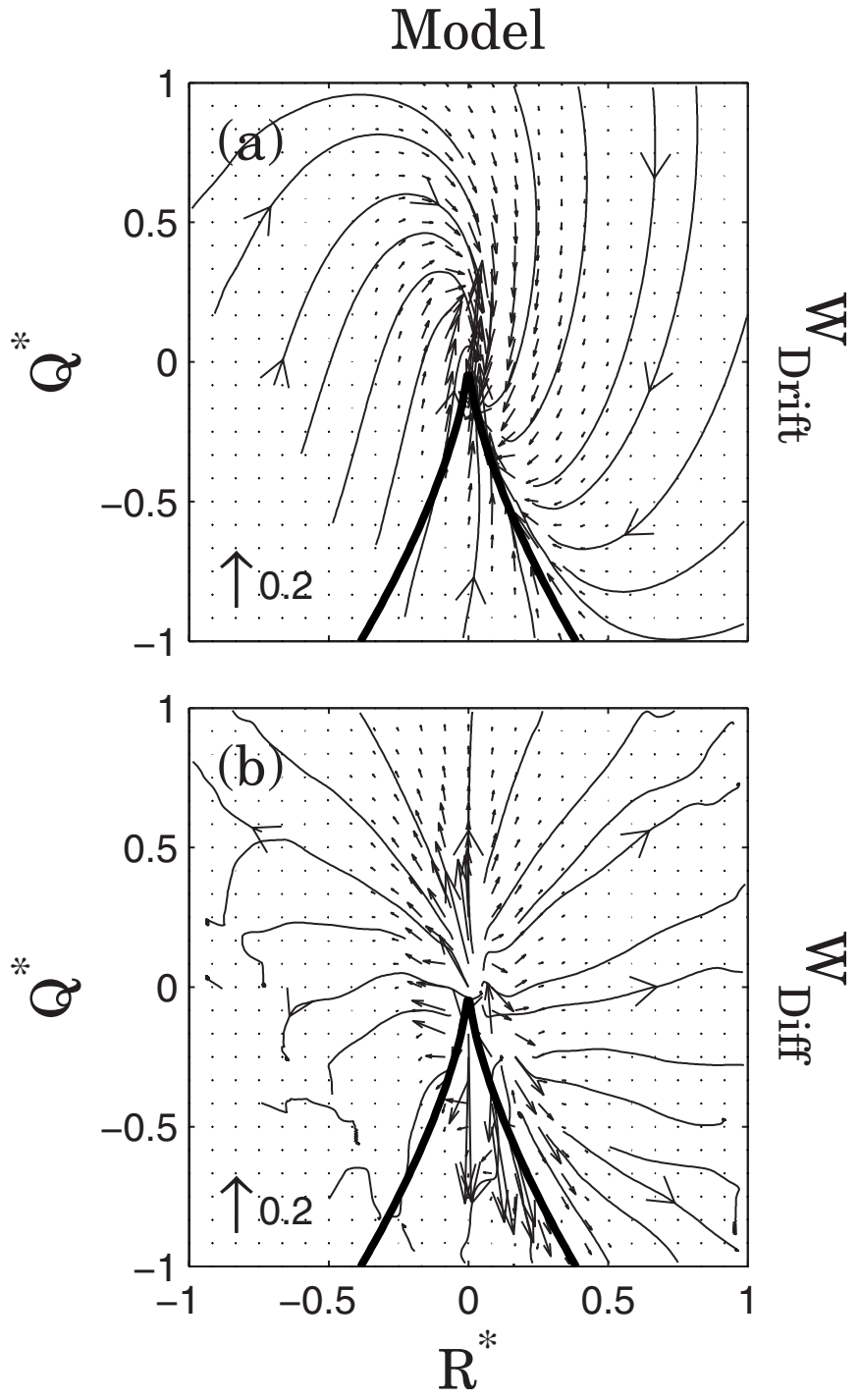

FIG. 10. Vector and streamline plots of the probability current for the model associated with the (a) drift coefficients and (b) diffusion coefficients [see text and Eq. (B9)]. The scale of the vectors is the same, reference is given in each figure, whose (nondimensionalized) magnitude is $2 \times 10^{-1}$.

We see from Eq. (B9) that one needs to add another term $\mathcal{W}_{\text {diff }}$ to the probability current $\mathcal{W}$ when dealing with a SDE. This term does not exist when dealing with a deterministic equation [Eq. (28)], although the gradient of the forcing entering in the Navier-Stokes equations for $\mathbf{A}$ [Eq. (1)] has been neglected. The total probability current $\mathcal{W}$ [Eq. (B9)] was displayed in Fig. 5(h). We would like now to display separately the probability current coming from the drift terms $\mathcal{W}_{\text {drift }}$ and the one generated by the diffusion coefficients $\mathcal{W}_{\text {diff. }}$ We represent in Fig. 10 the vector and streamline plots of the probability current for the model associated [Fig. 10(a)] with the drift coefficients $\mathcal{W}_{\text {drift }}$ and [Fig. 10(b)] with the diffusion coefficients $\mathcal{W}_{\text {diff }}$ [Eq. (B9)]. We recall that the total probability current is displayed in Fig. 5(h). We see first that $\mathcal{W}_{\text {drift }}$ and $\mathcal{W}_{\text {diff }}$ are of the same order of magnitude. The current $\mathcal{W}_{\text {drift }}$ associated with the deterministic part of the stochastic evolution [Eq. (B4)] goes toward the origin in a rotating motion: the dynamics is decaying. To compensate for this decay, the current $\mathcal{W}_{\text {diff }}$ associated with the stochastic forcing part of the evolution [Eq. (B4)] points outward away from the origin. The sum of these two, the total current displayed in Fig. 5(h), has a circular motion around the origin, consistent with a stationary process [i.e., $\partial \mathcal{P}(Q, R) / \partial t=-\nabla \cdot \mathcal{W} \approx 0]$.

${ }^{1}$ A. N. Kolmogorov, "The local structure of turbulence in incompressible viscous fluid for very large Reynolds numbers," Dokl. Akad. Nauk SSSR 30, 301 (1941) (in Russian) [English translation: Proc. R. Soc. London, Ser. A 434, 9 (1991)].

${ }^{2} \mathrm{H}$. Tennekes and J. L. Lumley, A First Course in Turbulence (MIT Press, Cambridge, MA, 1972).

${ }^{3}$ R. H. Kraichnan, "On Kolmogorov's inertial-range theories," J. Fluid Mech. 62, 305 (1974).

${ }^{4}$ A. S. Monin and A. M. Yaglom, Statistical Fluid Mechanics (MIT Press, Cambridge, MA, 1975).

${ }^{5}$ U. Frisch, Turbulence (Cambridge University Press, Cambridge, 1995).

${ }^{6}$ S. B. Pope, Turbulent Flows (Cambridge University Press, Cambridge, 2000).

${ }^{7}$ A. Tsinober, An Informal Introduction to Turbulence (Kluwer Academic, Dordrecht, 2001).

${ }^{8}$ J. Léorat, "Etude de la turbulence MHD homogène et isotrope et application à l'effet dynamo," Ph.D. thesis, Université Paris-VII, 1975.

${ }^{9} \mathrm{P}$. Vieillefosse, "Local interaction between vorticity and shear in a perfect incompressible fluid," J. Phys. (Paris) 43, 837 (1982).

${ }^{10} \mathrm{P}$. Vieillefosse, "Internal motion of a small element of fluid in an inviscid flow," Physica A 125, 150 (1984).

${ }^{11}$ B. J. Cantwell, "Exact solution of a restricted Euler equation for the velocity gradient tensor," Phys. Fluids A 4, 782 (1992).

${ }^{12}$ W. T. Ashurst, A. R. Kerstein, R. M. Kerr, and C. H. Gibson, "Alignment of vorticity and scalar gradient with strain rate in simulated Navier-Stokes turbulence," Phys. Fluids 30, 2343 (1987).

${ }^{13}$ B. J. Cantwell, "On the behavior of velocity gradient tensor invariants in direct numerical simulations of turbulence," Phys. Fluids A 5, 2008 (1993).

${ }^{14}$ T. S. Lund and M. M. Rogers, "An improved measure of strain state probability in turbulent flows," Phys. Fluids 6, 1838 (1994).

${ }^{15}$ A. Ooi, J. Martin, J. Soria, and M. S. Chong, "A study of the evolution and characteristics of the invariants of the velocity-gradient tensor in isotropic turbulence," J. Fluid Mech. 381, 141 (1999).

${ }^{16}$ F. van der Bos, B. Tao, C. Meneveau, and J. Katz, "Effects of small-scale turbulent motions on the filtered velocity gradient tensor as deduced from holographic PIV measurements," Phys. Fluids 14, 2457 (2002).

${ }^{17}$ B. Tao, J. Katz, and C. Meneveau, "Statistical geometry of subgrid-scale stresses determined from holographic particle image velocimetry measurements," J. Fluid Mech. 467, 35 (2002).

${ }^{18}$ B. W. Zeff, D. D. Lanterman, R. McAllister, R. Roy, E. J. Kostelich, and D. P. Lathrop, "Measuring intense rotation and dissipation in turbulent flows," Nature (London) 421, 146 (2003).

${ }^{19}$ B. Luthi, A. Tsinober, and W. Kinzelbach, "Lagrangian measurement of vorticity dynamics in turbulent flow," J. Fluid Mech. 528, 87 (2005).

${ }^{20}$ J. D. Gibbon and D. D. Holm, "Lagrangian particle paths and orthonormal quaternion frames," Nonlinearity 20, 1745 (2007).

${ }^{21}$ B. Castaing, Y. Gagne, and E. Hopfinger, "Velocity probability density functions of high Reynolds number turbulence," Physica D 46, 177 (1990).

${ }^{22}$ P. Kailasnath, K. R. Sreenivasan, and G. Stolovitzky, "Probability density of velocity increments in turbulent flows," Phys. Rev. Lett. 68, 2766 (1992).

${ }^{23}$ L. Chevillard, B. Castaing, E. Lévêque, and A. Arneodo, "Unified multifractal description of velocity increments statistics in turbulence: Intermittency and skewness," Physica D 218, 77 (2006).

${ }^{24}$ R. Benzi, L. Biferale, G. Paladin, A. Vulpiani, and M. Vergassola, "Multifractality in the statistics of the velocity gradients in turbulence," Phys. Rev. Lett. 67, 2299 (1991).

${ }^{25}$ M. Nelkin, "Multifractal scaling of velocity derivatives in turbulence," Phys. Rev. A 42, 7226 (1990).

${ }^{26}$ K. R. Sreenivasan and R. Antonia, "The phenomenology of small-scale turbulence," Annu. Rev. Fluid Mech. 29, 435 (1997).

${ }^{27}$ R. H. Kraichnan, "Models of intermittency in hydrodynamic turbulence," Phys. Rev. Lett. 65, 575 (1990). 
${ }^{28}$ R. H. Kraichnan, “Turbulent cascade and intermittency growth," Proc. R. Soc. London, Ser. A 434, 65 (1991).

${ }^{29}$ S. S. Girimaji and S. B. Pope, "A diffusion model for velocity gradients in turbulence," Phys. Fluids A 2, 242 (1990).

${ }^{30}$ M. Chertkov, A. Pumir, and B. I. Shraiman, "Lagrangian tetrad dynamics and the phenomenology of turbulence," Phys. Fluids 11, 2394 (1999).

${ }^{31}$ E. Jeong and S. S. Girimaji, "Velocity-gradient dynamics in turbulence: Effect of viscosity and forcing," Theor. Comput. Fluid Dyn. 16, 421 (2003).

${ }^{32} \mathrm{Y}$. Li and C. Meneveau, "Origin of non-Gaussian statistics in hydrodynamic turbulence," Phys. Rev. Lett. 95, 164502 (2005).

${ }^{33} \mathrm{Y}$. Li and C. Meneveau, "Intermittency trends and Lagrangian evolution of non-Gaussian statistics in turbulent flow and scalar transport," J. Fluid Mech. 558, 133 (2006).

${ }^{34}$ L. Chevillard and C. Meneveau, "Lagrangian dynamics and statistical geometric structure of turbulence," Phys. Rev. Lett. 97, 174501 (2006).

${ }^{35}$ L. Chevillard and C. Meneveau, "Intermittency and universality in a Lagrangian model of velocity gradients in three-dimensional turbulence," C. R. Mec. 335, 187 (2007).

${ }^{36}$ L. Biferale, L. Chevillard, C. Meneveau, and F. Toschi, "Multi-scale model of gradient evolution in turbulent flows," Phys. Rev. Lett. 98, 214501 (2007)

${ }^{37}$ M. S. Chong, A. E. Perry, and B. J. Cantwell, "A general classification of three-dimensional flow fields," Phys. Fluids A 2, 765 (1990).

${ }^{38} \mathrm{~S}$. B. Pope and Y. L. Chen, "The velocity-dissipation probability density function model for turbulent flows," Phys. Fluids A 2, 1437 (1990).

${ }^{39}$ A. Naso and A. Pumir, "Scale dependence of the coarse-grained velocity derivative tensor structure in turbulence," Phys. Rev. E 72, 056318 (2005).

${ }^{40}$ A. Naso, A. Pumir, and M. Chertkov, "Statistical geometry in homogeneous and isotropic turbulence," J. Turbul. 8, 39 (2007).

${ }^{41}$ B. Cantwell, "A singularity-free model of the local velocity gradient and acceleration gradient structure of turbulent flow," in Tubes, Sheets and Singularities in Fluid Dynamics, Proceedings of the IUTAM Symposium, Zakopane, Poland, 2002, edited by K. Bajer and H. K. Moffatt (Kluwer Academic, Dordrecht, 2001).

${ }^{42} \mathrm{P}$. Constantin, "An Eulerian-Lagrangian approach to the Navier-Stokes equations," Commun. Math. Phys. 216, 663 (2001).

${ }^{43}$ A. Majda and A. Bertozzi, Vorticity and Incompressible Flow (Cambridge University Press, Cambridge, 2002).

${ }^{44}$ J. D. Dollard and C. N. Friedman, Product Integration (Addison-Wesley, London, 1979).

${ }^{45}$ C. Itzykson and J.-B. Zuber, Quantum Field Theory (McGraw-Hill, New York, 1980).
${ }^{46}$ G. Falkovich, K. Gawędzki, and M. Vergassola, "Particles and fields in fluid turbulence," Rev. Mod. Phys. 73, 913 (2001).

${ }^{47} \mathrm{C}$. Truesdell and W. Noll, The Non-Linear Field Theories of Mechanics (Springer-Verlag, Berlin, 1992).

${ }^{48}$ S. S. Girimaji and S. B. Pope, "Material element deformation in isotropic turbulence," J. Fluid Mech. 220, 427 (1990).

${ }^{49}$ K. K. Nomura and G. K. Post, "The structure and dynamics of vorticity and rate of strain in incompressible homogeneous turbulence," J. Fluid Mech. 377, 65 (1998).

${ }^{50} \mathrm{~K}$. Ohkitani, "Eigenvalue problems in three-dimensional Euler flows," Phys. Fluids A 5, 2570 (1993).

${ }^{51} \mathrm{~K}$. Ohkitani and S. Kishiba, "Nonlocal nature of vortex stretching in an inviscid fluid," Phys. Fluids 7, 411 (1995).

${ }^{52}$ P. E. Kloeden and E. Platen, Numerical Solution of Stochastic Differential Equations (Springer, Berlin, 1999).

${ }^{53}$ P. K. Yeung, S. B. Pope, and B. L. Sawford, "Reynolds number dependence of Lagrangian statistics in large numerical simulations of isotropic turbulence," J. Turbul. 7, 58 (2006).

${ }^{54}$ C. Rosales and C. Meneveau, "A minimal multiscale Lagrangian map approach to synthesize non-Gaussian turbulent vector fields," Phys. Fluids 18, 075104 (2006).

${ }^{55}$ G. Eyink, "Local energy flux and the refined similarity hypothesis," J. Stat. Phys. 78, 335 (1995).

${ }^{56} \mathrm{~V}$. Borue and S. A. Orszag, "Local energy flux and subgrid-scale statistics in three-dimensional turbulence," J. Fluid Mech. 366, 1 (1998).

${ }^{57} \mathrm{C}$. Meneveau and J. Katz, "Scale-invariance and turbulence models for large-eddy simulation," Annu. Rev. Fluid Mech. 32, 1 (2000).

${ }^{58}$ H. Risken, The Fokker-Planck Equation (Springer-Verlag, Berlin, 1984).

${ }^{59}$ B. Galanti, J. D. Gibbon, and M. Heritage, "Vorticity alignment results for the three-dimensional Euler and Navier-Stokes equations," Nonlinearity 10, 1675 (1997)

${ }^{60}$ J. D. Gibbon, D. D. Holm, R. M. Kerr, and I. Roulstone, "Quaternions and particle dynamics in the Euler fluid equations," Nonlinearity 19, 1969 (2006).

${ }^{61}$ A. Tsinober, L. Shtilman, A. Sinyavskii, and H. Vaisburd, "Vortex stretching and enstrophy generation in numerical and laboratory turbulence," in Small-Scale Structures in Three-Dimensional Hydrodynamic and Magnetohydrodynamic Turbulence, Proceedings of a Workshop, Nice, France, edited by M. Meneguzzi, A. Pouquet, and P.-L. Sulem (Springer, Berlin, 1995), pp. 9-16.

${ }^{62}$ C. Kalelkar, "Statistics of pressure fluctuations in decaying isotropic turbulence," Phys. Rev. E 73, 046301 (2006).

${ }^{63}$ B. Øksendal, Stochastic Differential Equations (Springer-Verlag, Berlin, 2003). 